\title{
Metabolism and Mass Balance of the Novel Nonsteroidal Androgen Receptor Inhibitor Darolutamide in Humans ${ }^{\mathbb{\$}}$
}

\author{
Päivi Taavitsainen, Olaf Prien, ${ }^{1}$ Marja Kähkönen, Michael Niehues, ${ }^{1}$ Timo Korjamo, \\ Karsten Denner, Pirjo Nykänen, Annamari Vuorela, Natalia A. Jungmann, \\ Clemens-Jeremias von Bühler, Mikko Koskinen, Christian Zurth, and @ Hille Gieschen
}

Orion Corporation Orion Pharma, Turku, Finland (P.T.); Bayer AG, Berlin, Germany (O.P., M.N., K.D., C.Z., H.G.); Orion Corporation Orion Pharma, Espoo, Finland (M.K., T.K., P.N., A.V., M.K.); and Bayer AG, Wuppertal, Germany (N.A.J., C.-J.v.B.)

Received November 10, 2020; accepted March 10, 2021

\section{ABSTRACT}

The biotransformation and excretion of darolutamide were investigated in a phase I study. Six healthy male volunteers received a single dose of $300 \mathrm{mg}{ }^{14} \mathrm{C}$-darolutamide as an oral solution in the fasted state. Plasma, urine, and feces samples were analyzed for mass balance evaluation by liquid scintillation counting (LSC). Metabolite profiling and identification were determined using liquid chromatography mass-spectrometry with off-line radioactivity detection using LSC. Complete mass balance was achieved, with mean radioactivity recovery of $95.9 \%$ within 168 hours $(63.4 \%$ in urine, $32.4 \%$ in feces). The administered $1: 1$ ratio of $(S, R)$ - and $(S, S)$ darolutamide changed to approximately $1: 5$, respectively, in plasma. Darolutamide and the oxidation product, keto-darolutamide, were the only components quantifiable by LSC in plasma, accounting for $\mathbf{8 7 . 4} \%$ of total radioactivity, with a 2.1 -fold higher plasma exposure

This work was supported by Bayer AG and Orion Corporation Orion Pharma. Clemens-Jeremias von Bühler and Natalia Jungmann report employment by Bayer. Hille Gieschen, Karsten Denner, and Christian Zurth report employment by and stock ownership in Bayer. Michael Niehues and Olaf Prien are employed by NUVISAN, Inc., but were employees of Bayer, and Dr. Prien held stock in Bayer at the time that the work was performed. Marja Kähkönen, Pirjo Nykänen, Päivi Taavitsainen, and Annamari Vuorela report employment by Orion. Timo Korjamo and Mikko Koskinen report employment by and stock ownership in Orion.

Availability of the data underlying this publication will be determined later according to Bayer's commitment to the EFPIA/PhRMA "Principles for responsible clinical trial data sharing." This pertains to scope, time point, and process of data access.

As such, Bayer commits to sharing upon request from qualified scientific and medical researchers patient-level clinical trial data, study-level clinical trial data, and protocols from clinical trials in patients for medicines and indications approved in the United States and European Union as necessary for conducting legitimate research. This applies to data on new medicines and indications that have been approved by the United States and European Union regulatory agencies on or after January 1, 2014.

Interested researchers can use www.clinicalstudydatarequest.com to request access to anonymized patient-level data and supporting documents from clinical studies to conduct further research that can help advance medical science or improve patient care. Information on the Bayer criteria for listing studies and other relevant information is provided in the Study sponsors section of the portal.

Data access will be granted to anonymized patient-level data, protocols, and clinical study reports after approval by an independent scientific review panel. Bayer is not involved in the decisions made by the independent review panel. Bayer will take all necessary measures to ensure that patient privacy is safeguarded.

${ }^{1}$ Current affiliation: Nuvisan ICB GmbH, Berlin, Germany.

https://doi.org/10.1124/dmd.120.000309.

S This article has supplemental material available at dmd.aspetjournals.org. for keto-darolutamide. Aside from darolutamide, the most prominent metabolites in urine were 0 -glucoronide $(\mathrm{M}-7 \mathrm{a} / \mathrm{b})$ and $N$-glucuronide (M-15a/b), as well as pyrazole sulfates (M-29, $M-24)$ and glucuronides (M-21, $\mathrm{M}-22)$ resulting from oxidative cleavage of the parent. The darolutamide diastereomers were mainly detected in feces. In vitro assays showed that darolutamide metabolism involves a complex interplay between oxidation and reduction, as well as glucuronidation. Interconversion of the diastereomers involves oxidation to keto-darolutamide, primarily mediated by CYP3A4, followed by reduction predominantly catalyzed by cytosolic reductase(s), with aldo-keto reductase $1 \mathrm{C3}$ playing the major role. The latter reaction showed stereoselectivity with preferential formation of $(S, S)$-darolutamide.

\section{SIGNIFICANCE STATEMENT}

The metabolism and excretion of darolutamide in humans revealed that oxidation (CYP3A4) and glucuronidation (UGT1A9, UGT1A1) were the main metabolic routes of elimination. Direct excretion also contributed to overall clearance. The two pharmacologically equipotent diastereomers of darolutamide interconvert primarily via oxidation to the active metabolite keto-darolutamide, followed by reduction predominantly by cytosolic reductase(s). The latter reaction showed stereoselectivity with preferential formation of $(S, S)$ darolutamide. Data indicate a low drug-drug interaction potential of darolutamide with inducers or inhibitors of metabolizing enzymes.

\section{Introduction}

Darolutamide (Nubeqa, formerly ODM-201) is a potent and selective nonsteroidal androgen receptor inhibitor developed as an immediaterelease, oral, 300-mg tablet and is approved for the treatment of nonmetastatic castration-resistant prostate cancer (nmCRPC) (https:// www.accessdata.fda.gov/drugsatfda_docs/label/2019/212099Orig1s000lbl. pdf; https://www.ema.europa.eu/documents/product-information/nubeqa-eparproduct-information_en.pdf; https://www.bayer.com/sites/default/files/ 2021-02/Bayer-Annual-Report-2020.pdf) based on significantly improved survival outcomes versus placebo in men with nmCRPC receiving androgen deprivation therapy (Fizazi et al., 2019, 2020). Darolutamide is a flexible pyrazole with polar substitutions and is structurally distinct from other androgen receptor inhibitors (Moilanen et al., 2015). Darolutamide is a 1:1 mixture of the two diastereomers, $(S, R)$-darolutamide and $(S, S)$-darolutamide, which interconvert via the major metabolite ketodarolutamide (Fig. 1) (Nykänen et al., 2020; Taavitsainen et al., 2020), 
<smiles>C[C@H](Cn1ccc(-c2ccc(C#N)c(Cl)c2)n1)NC(=O)c1cc([Ge])[nH]n1</smiles>

(S,S)-darolutamide<smiles>C[C@H](Cn1ccc(-c2ccc(C#N)c(Cl)c2)n1)NC(=O)c1cc([C@@H](C)O)[nH]n1</smiles>

$(S, R)$-darolutamide

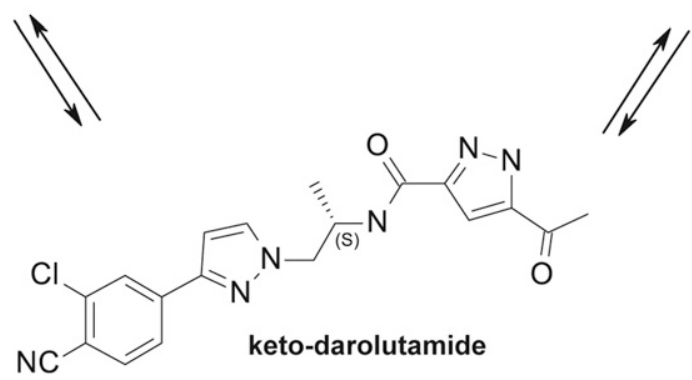

Fig. 1. Darolutamide diastereomers, $(S, R)$-darolutamide and $(S, S)$-darolutamide, interconvert via the major metabolite keto-darolutamide.

and all three compounds show similar pharmacological activity in vitro (Moilanen et al., 2015; Sugawara et al., 2019).

The present phase I study was conducted to fully characterize the pharmacokinetics, metabolic pathways, and excretion routes of darolutamide, determined after single administration of darolutamide to healthy male volunteers. The investigation of the pharmacokinetic characteristics of darolutamide after single intravenous and oral administration will be published separately. The evaluation of the metabolism and excretion of ${ }^{14} \mathrm{C}$-darolutamide in humans is reported herein. In addition, complementary in vitro studies conducted in human biomaterials to identify the relevant enzymes involved in the biotransformation of darolutamide and its major metabolite, keto-darolutamide, are presented.

\section{Methods}

\section{Phase I Study}

Study Design. The absorption, metabolism, routes of excretion, and mass balance of darolutamide and its metabolites were evaluated in a single-center, open-label phase I study performed by Quotient Clinical (Nottingham, UK) in six healthy male volunteers. The study was conducted in accordance with Good Clinical Practice Guidelines (https://database.ich.org/sites/default/ files/E6_R2_Addendum.pdf). An independent ethics committee reviewed and approved the study protocol, and all subjects provided written, informed consent.

Study Population. Six healthy male subjects (five White, one Black) were enrolled in part 2 of the study and were included in the analyses. At baseline, mean age was 53.0 years (range 50.0-56.0 years), mean weight was $82.7 \mathrm{~kg}$ (range $63.7-91.0 \mathrm{~kg}$ ), and mean body mass index was $27.1 \mathrm{~kg} / \mathrm{m}^{2}$ (range $24.0-30.4 \mathrm{~kg} / \mathrm{m}^{2}$ ). None of the subjects were current smokers or consumed $>14$ units of alcohol per week. Subjects were discharged from the clinic on day 8,168 hours postdose, having met the radioactivity release criteria, i.e., mass balance recovery of $>90 \%$ had been achieved or two consecutive days showed $<1 \%$ recovery.

Study Medication. On day 1 , subjects received a single oral dose of approximately $300 \mathrm{mg}{ }^{14} \mathrm{C}$-darolutamide (actual range 301.3-302.2 mg) as an oral solution, containing on average $5.4-\mathrm{MBq}{ }^{14} \mathrm{C}$, after fasting for $\geq 10$ hours overnight; the fasted state was maintained until approximately 4 hours postdose. The position of the ${ }^{14} \mathrm{C}$ - label was at the phenyl moiety of darolutamide [the ${ }^{14} \mathrm{C}$-labeling of darolutamide and reference compounds are described in detail elsewhere (Taavitsainen et al., 2020)]. Dosimetry calculations were provided by Public Health England Centre for Radiation, Chemical and Environmental Hazards (Chilton, UK) according to local regulations. The associated radiation exposure fell within International Commission on Radiologic Protection (1992) guidelines for category IIa studies $(0.1-1.0 \mathrm{mSv})$.

Sample Collection. Blood samples for evaluation of pharmacokinetic parameters, total radioactivity, and metabolite profiling and identification in plasma were collected predose and at $0.25,0.5,1$, $1.5,2,3,4,5,6,8,12,16,24,48,72,96,120,144$, and 168 hours postdose. Whole blood was centrifuged to obtain the plasma fraction. In radioactive metabolite profiling, individual plasma samples were analyzed up to 48 hours postdose. For profiling and identification of metabolites that lacked the radioactive label because of metabolism, plasma samples $(50 \mu \mathrm{L}$ each) were pooled by individual time points across the subjects for analysis by liquid chromatography-mass spectrometry (LC-MS). Urine samples for determination of total radioactivity and metabolite profiling were collected over the following intervals: predose, 0-12 hours, and 12-24 hours after drug administration, and then for each 24-hour period up to 168 hours postdose. Each subject's urine, collected in individual polypropylene containers during these intervals, was pooled prior to analysis to produce a single bulk sample for each interval per subject. Fecal samples for total radioactivity and metabolic profiling were collected predose and every 24 hours up to 168 hours postdose. Each subject's samples were pooled per 24-hour 
time period prior to analysis of total radioactivity. All excreta samples were stored at least at $-20^{\circ} \mathrm{C}$ until further analysis.

Sample Preparation for Metabolite Profiling and Identification. Full details of equipment and materials for metabolite profiling and quantitation are provided in Supplemental Table 1. Plasma, urine, and fecal samples were prepared for liquid chromatography (LC) analysis with off-line radioactivity detection (method B, Supplemental Table 2). Plasma samples for radioactive metabolite profiling $(0.25-0.5 \mathrm{~mL})$ were treated with equal volumes of ammonium formate $(25 \mathrm{mM})$ containing $5 \%$ acetonitrile at $\mathrm{pH} 4$ (solvent $\mathrm{A}$ ) and $1 \mathrm{~mL}$ of acetonitrile and chilled for 10 minutes. The plasma samples were homogenized, and the proteins were separated from the supernatant by centrifugation (10 minutes, $\left.3000 \mathrm{~g}, 4^{\circ} \mathrm{C}\right)$. Subsequently, the supernatants were evaporated to dryness and dissolved in solvent A (method B, Supplemental Table 2) prior to analysis. Individual urine samples (per subject and collection period) were mixed thoroughly and diluted with solvent A (1:1) prior to analysis (method B, Supplemental Table 2). Fecal samples were wet homogenized before analysis of total ${ }^{14} \mathrm{C}$ radioactivity and metabolite profiling. For metabolite profiling, homogenized aliquots $(0.35-0.50 \mathrm{~g})$ were subsequently extracted with acetonitrile $(5 \mathrm{~mL})$ for 1 hour and then centrifuged, and the supernatants were concentrated by evaporation to 1-4 mL and then diluted 1:1 with solvent A before analysis (method B, Supplemental Table 2). The mean recovery of total radioactivity extraction for the different sampling time points amounted to $98.2 \%$. For profiling and identification of metabolites that lacked the radioactive label because of cleavage of the central amide bond of darolutamide, samples were prepared for LC-MS analysis (method C, Supplemental Table 2). Plasma samples were diluted with ammonium acetate buffer, $\mathrm{pH} 4$, and proteins were precipitated using acetonitrile $(100 \mu \mathrm{L}$ of plasma, $100 \mu \mathrm{L}$ of solvent A, and $400 \mu \mathrm{L}$ of acetonitrile). Samples were then centrifuged, the supernatant was filtered into protein precipitation plates and centrifuged, and then the filtrate was concentrated. Samples were diluted with ammonium acetate buffer, $\mathrm{pH} 4$, centrifuged, and the supernatant was transferred to vials for LC-MS analysis. Urine and fecal samples were prepared using the same procedure as described for radiolabeled metabolites.

Fecal samples were also prepared to investigate the stability of ketodarolutamide ex vivo by mimicking the anaerobic microbial environment of the gut. Under anaerobic conditions, ${ }^{14} \mathrm{C}$-keto-darolutamide ( $1 \mathrm{mM}$ in acetonitrile) was added to a slurry of fresh human feces in degassed water and incubated for 24 hours at $37^{\circ} \mathrm{C}$. The incubation mixture was stopped by adding acetonitrile and centrifuged, and the supernatant was subjected to LC with off-line radioactivity detection (method B, Supplemental Table 2).

Determination of Total Radioactivity. Total radioactivity concentrations in body fluids and excreta, and mass balance calculations were determined by Quotient Bioresearch Ltd (Rushden, UK) using liquid scintillation counting (LSC) in a liquid scintillation spectrometer, TriCarb 2900 TR Liquid Scintillation Analyzer (Perkin Elmer, Shelton), with automatic quench correction by the external standard channel ratio method at $13^{\circ} \mathrm{C}$ using Atomlight as scintillation cocktail. Lower limits of quantitation for total ${ }^{14} \mathrm{C}$ radioactivity were $30.57 \mathrm{ng} \mathrm{eq} / \mathrm{mL}$ in plasma, $7.17 \mathrm{ng} \mathrm{eq} / \mathrm{mL}$ in urine, and $64.32 \mathrm{ng} \mathrm{eq} / \mathrm{mL}$ in feces.

Metabolite Profiling in Human Plasma, Urine, and Feces. Metabolic profiles were investigated by analytical LC on reversed phase columns, followed by off-line radioactivity detection (method B, Supplemental Table 2). Radioactivity was detected using a TopCount NXT Microplate Scintillation and Luminescence Counter (Perkin Elmer, Boston). In the radiochromatograms obtained, the drug diastereomers were not separated and were analyzed as the sum of both. Semiquantitative LC-MS analysis was performed to measure metabolites lacking the radioactive label (method C, Supplemental Table 2); their concentrations were calculated based on a calibration curve prepared from respective reference standards. The synthesis of the respective metabolite reference standards is described in the Supplemental Methods 1.

Determination of Darolutamide and Keto-Darolutamide. Concentrations of $(S, R)$-darolutamide, $(S, S)$-darolutamide, and ketodarolutamide in plasma and concentrations of the diastereomers in urine were determined using a validated (plasma) or qualified (urine) highperformance liquid chromatography-tandem mass spectrometry (LCMS/MS) analytical method at PRA Health Sciences (Assen, The Netherlands) (method A, Supplemental Table 2). The lower limit of quantification for keto-darolutamide and both diastereomers in plasma and urine was $5 \mathrm{ng} / \mathrm{mL}$. Concentrations of darolutamide were calculated as the sum of the two diastereomers, $(S, R)$ - and $(S, S)$-darolutamide. Quality criteria of these bioanalytical methods are described in detail in the Supplemental Methods 2 and Supplemental Table 3. Measurement of $(S, R)$-darolutamide and $(S, S)$-darolutamide in samples from human feces was done by LC-MS analysis (method D, Supplemental Table 2).

Pharmacokinetic Evaluation. Pharmacokinetic analysis of the plasma concentration versus time data as well as from metabolic profiles was performed using appropriate noncompartmental techniques to obtain estimates of parameters including exposure [area under the concentration-time curve $\left.(\mathrm{AUC})_{0-\text { inf }}, \mathrm{AUC}_{0-\text { tlast }}\right], C_{\max }$, time when maximum plasma concentration is observed, and apparent terminal half-life $\left(t_{1 / 2}\right)$. For urine, the amount excreted $\left(\mathrm{A}_{\mathrm{e}}\right)$, the renal clearance, and the excretion $t_{1 / 2}$ were calculated.

In addition, renal clearance of darolutamide was further investigated. The estimated glomerular filtration rate (eGFR) in the individual subjects was estimated by applying the Chronic Kidney Disease Epidemiology Collaboration formula (Levey et al., 2009) using individual physical information of the subjects (i.e., age, sex, and ethnic origin) and a normalized value for the body surface area of $1.73 \mathrm{~m}^{2}$. This individual eGFR was used as a marker for passive glomerular filtration and tubular secretion. The renal clearance of $(S, S)$ - and $(S, R)$-darolutamide was calculated based on drug amounts excreted and the unbound plasma exposure $\left[\mathrm{AUC}_{0-\mathrm{inf}}\right.$ (Brater, 2002).

\section{In Vitro Studies}

In Vitro Metabolic Profiling and Substrate Characteristics. Several studies were conducted to investigate the biotransformation of darolutamide and its diastereomers in vitro in human cryopreserved suspension hepatocytes (from different donors), subcellular liver preparations (pool), and recombinant enzymes. Full details of materials for the in vitro studies are provided in Supplemental Table 1. Depending on the purpose of the experiment, the starting material was darolutamide $\left({ }^{14} \mathrm{C}\right.$-labeled or nonlabeled), its diastereomers, or keto-darolutamide $\left({ }^{14} \mathrm{C}\right.$-labeled or nonlabeled), and the initial concentration range was 1-50 $\mu \mathrm{M}$, with $1 \mu \mathrm{M}$ used in most experiments. The total incubation time varied between different experiments. Several samples were typically withdrawn during incubation, which were quenched with acetonitrile and stored cold until analysis. After sample preparation using appropriate techniques, the supernatants were subjected to LC with LSC for radioactivity detection and LC-MS or LC-MS/MS analysis. For darolutamide, both nonchiral and chiral analyses were used. When both diastereomers were analyzed separately, total darolutamide was calculated as the sum of these two measured concentrations.

${ }^{14} \mathrm{C}$-Darolutamide was incubated in suspension hepatocytes from several donors for up to 4 hours to obtain quantitative in vitro metabolite profiles. Hepatocyte incubations in the presence of the CYP3A4 inhibitor itraconazole were performed to evaluate the proposed main metabolic pathway. To quantify substrate depletion and metabolite formation, ${ }^{14} \mathrm{C}$-darolutamide was incubated in human liver microsomes 
(HLM) and a panel of 21 recombinant human P450 isoforms (Supplemental Table 1). CYP3A4-specific inhibitors (ketoconazole, cobicistat) were also applied in microsomal incubations. Additionally, incubations of human liver cytosol (HLC) and recombinant enzymes under different $\mathrm{pH}$ and cofactor conditions were used to evaluate the contribution of non-P450 enzymes in darolutamide oxidation.

Keto-darolutamide $\left({ }^{14} \mathrm{C}\right.$-labeled or nonlabeled) metabolism was studied in incubations of hepatocytes, HLM, HLC, and several recombinant enzymes [P450, aldo-keto reductase (AKR)]. The CYP3A4 inhibitor itraconazole and AKR inhibitors flufenamic acid (AKR1C1-4) and EM1404 (AKR1C3; synthesized at Bayer AG, Germany) were included in selected incubations. Both darolutamide diastereomers and other metabolites were analyzed from these incubations.

The interconversion of darolutamide diastereomers via ketodarolutamide was investigated in suspension hepatocytes with $(S, R)$ darolutamide, $(S, S)$-darolutamide, or keto-darolutamide as starting material. In this context, the AKR1C3 inhibitor BAY 1128688 (synthesized at Bayer AG, Germany) and the CYP3A4 inhibitor cobicistat were used to evaluate the contribution of these pathways. Diastereomer-specific analytical methods were applied to these incubations.

To assess the role of glucuronidation in darolutamide biotransformation, darolutamide and both diastereomers were incubated with human liver, kidney, and intestinal microsomes and a panel of 13 recombinant uridine-diphosphate glucuronosyltransferase (UGT) isoforms (Supersomes) (Supplemental Table 1). Incubations were fortified with uridine 5 '-diphospho-glucuronic acid, $50 \mu \mathrm{g}$ alamethicin per milligram microsomal protein, and $\mathrm{MgCl}_{2}(5 \mathrm{mM})$. Experiments were performed in the presence and in the absence of enzyme-selective inhibitors to evaluate the relevance of specific pathways. To confirm the relative contribution of UGT1A1 and UGT1A9 in the overall glucuronidation process of darolutamide, the relative activity factor (RAF) method was used (Gibson et al., 2013). In short, RAFs were determined based on propofol (UGT1A9) and estradiol (UGT1A1) glucuronidation activities in HLM relative to the activities in recombinantly expressed UGT1A9 and UGT1A1, respectively. Relative contribution was calculated by applying RAF values to the glucuronidation activity of $(S, R)$ - and $(S, S)$ darolutamide determined in microsomes from baculovirus-transfected insect cells expressing human UGT isoforms.

For correlation analysis, HLM from 22 individual donors were characterized by incubation with the UGT isoform-selective substrates $\beta$-estradiol (UGT1A1), propofol (UGT1A9), $R$-flurbiprofen (UGT2B7), levomedetomidine (UGT2B10), and $S$-oxazepam (UGT2B15). The glucuronidation activities were correlated with the formation rates of M-7a/b and M-15a/b (substrate BAY 1841788) in the respective panel of microsomes.

Suppliers of the materials used in in vitro assays are listed in Supplemental Table 1.

Quantification of Darolutamide and Metabolites In Vitro. Metabolite profiles (radiochromatograms) were obtained using LC with radioactivity detection, with generation of metabolite pattern and integration of individual peaks (method F, Supplemental Table 4). LC-MS methods were applied for quantification of darolutamide and keto-darolutamide, chromatographic separation of the diastereomers, and quantitation of $(S, R)$ - and $(S, S)$-darolutamide. Individual conditions of the employed methods (e.g., stereoselective LC) were dependent on the analytical question. For evaluation of the role of glucuronidation in darolutamide biotransformation, metabolites were quantified using LCMS/MS methodology, and radioactivity was measured using LSC (method H, Supplemental Table 4). Bioanalytical methods are summarized in Supplemental Table 4.

Metabolite Identification. Chemical structures of the metabolites were identified or elucidated by LC-MS/MS based on retention times, exact mass, product ion spectra, and molecular formula and by comparison with authentic metabolite standards (M-1, M-26, M-30, M-32, M-33, and M-36). Structural proposals based on mass spectra interpretation were generated with a QExactive Plus high-resolution mass spectrometer (method B, Supplemental Table 2) using an electrospray ionization source and applying high collisional dissociation experiments to render product ion spectra. Electrospray ion voltage analysis was performed in both positive and negative ionization modes. In addition, in vitro samples of ${ }^{14} \mathrm{C}$-darolutamide in hepatocytes and recombinant P450 enzymes for metabolite profiling were analyzed by methods $\mathrm{F}$ and $\mathrm{G}$, respectively (Supplemental Table 4). Furthermore, metabolites M-7a, M-15a, M-21, and M-22 were isolated from urine and characterized by NMR.

Isolation and NMR Identification of Metabolites M-7a, M-15a, $\mathbf{M - 2 1}$, and M-22. Selected metabolites were isolated from pooled human urine (day 1, 0-12 hours). Urine was diluted with an equal volume of $0.1 \%$ formic acid and applied onto a C18 solid phase extraction (SPE) cartridge. Washing and elution was performed with acetonitrile $(\mathrm{ACN}) / 0.2 \%$ formic acid mixture by gradually increasing the solvent component up to $100 \% \mathrm{ACN}$. The fractions with the highest radioactivity were concentrated and subsequentially applied onto a $\mathrm{C} 18$ E SPE cartridge for further purification. Again, the SPE fractions with the highest radioactivity were retained for metabolite isolation by highperformance LC with a diode-array detector using a preparative column (Nucleodur C18 Gravity, $250 \times 21 \mathrm{~mm}, 5 \mu \mathrm{m}$ ). Using an isocratic elution with ACN/50 mM ammonium acetate (78:22, v/v) as mobile phase (flow: $20 \mathrm{~mL} / \mathrm{min}$ ), two fractions were collected containing respective diastereomeric mixtures of M-7a and M-7b $(S, S: S, R=92: 8)$ and M-15a and M-15b $(S, S: S, R=87: 13)$. Further purification of M-7a and $\mathrm{M}-15 \mathrm{a}$ was achieved by additional preparative high-performance LC with a diode-array detector using isocratic elution with ACN/ 0.2\% formic acid (M-7a: 73:27 [v/v] and M15a: 75:25 [v/v]). M-21 and M-22 were isolated from different SPE fractions using a gradient elution with ACN/0.2\% formic acid (20:80 at 0 minutes, 80:20 at 28 minutes). Purified fractions were then concentrated until dry under reduced pressure. Approximately $1 \mathrm{mg}$ of each isolated metabolite was dissolved in $\mathrm{CD}_{3} \mathrm{CN} / \mathrm{D}_{2} \mathrm{O}(70: 30 \mathrm{v} / \mathrm{v})$ for NMR on a $600-\mathrm{MHz}$ Bruker Ultrashield Magnet operating with an Avance III Bruker console using a 5-mm QNP Cryo Probe. ${ }^{1} \mathrm{H}$ spectra were recorded at $600.1 \mathrm{MHz}$, and ${ }^{13} \mathrm{C}$ spectra were recorded at $150.9 \mathrm{MHz}$. The residual $\mathrm{CD}_{3} \mathrm{CN}$ signal was used for spectral reference. For structural elucidation, ${ }^{1} \mathrm{H},{ }^{13} \mathrm{C}$, distortionless enhancement by polarization transfer 90 and 135, correlated spectroscopy, heteronuclear multiple-bond correlation, and heteronuclear singlequantum correlation spectra were recorded and analyzed.

\section{Results}

\section{Phase I Study}

Darolutamide Pharmacokinetics. After administration of a single dose of $300 \mathrm{mg}$ darolutamide as an oral solution, plasma concentrations were quantifiable (by method A in Supplemental Table 2) at the first time point ( 0.25 hours postdose), and $C_{\max }$ was reached after 1.5 hours (range 1.0-3.0 hours) (Fig. 2; Table 1). Thereafter, plasma concentrations declined in a biphasic manner with a terminal $t_{1 / 2}$ of 10.6 hours. Darolutamide was administered as a 1:1 mixture of the two diastereomers, $(S, R)$-darolutamide and $(S, S)$-darolutamide. In vivo, the ratio of drug diastereomers changed to 0.185 (approximately 1:5), based on the plasma $\mathrm{AUC}_{0-\text { inf }}$ data, which were determined separately for both diastereomers in the LC-MS/MS bioanalytical determination (Table 1; method A in Supplemental Table 2). Mean terminal $t_{1 / 2}$ for $(S, R)$ darolutamide was shorter than for $(S, S)$-darolutamide.

Urinary and Fecal Excretion of Darolutamide and Its Metabolites. After administration of $300 \mathrm{mg}{ }^{14} \mathrm{C}$-darolutamide, excretion of 


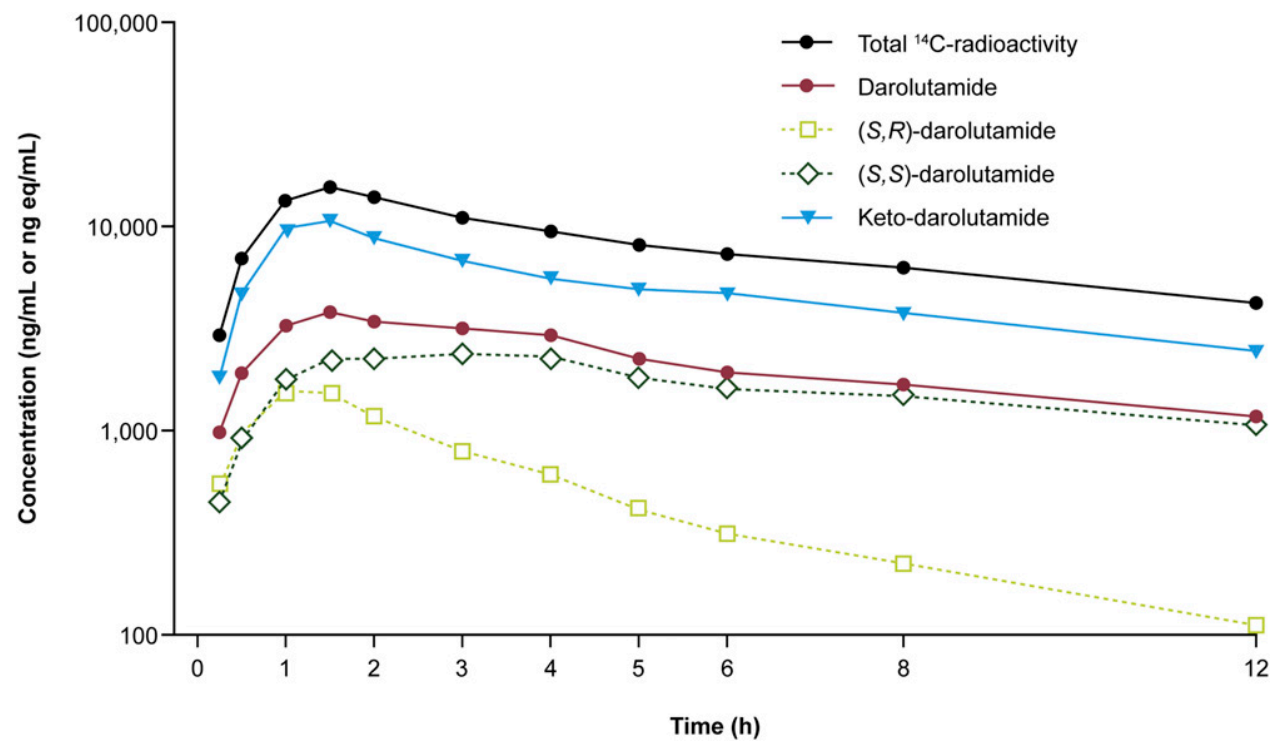

Fig. 2. Mean concentration-time profiles in plasma for darolutamide, $(S, S)$-darolutamide, $(S, R)$-darolutamide, and keto-darolutamide after a single dose of darolutamide $(300 \mathrm{mg})$ as an oral solution ( $n=6$ healthy male subjects).

radioactivity was rapid and relatively consistent across subjects. Mean recovery of $95.9 \%$ (range $92.6 \%-98.1 \%$ ) was reached by the end of the sampling period (168 hours), indicating complete mass balance (Fig. 3A); $63.4 \%$ (range $59.5 \%-68.9 \%$ ) of total radioactivity was recovered in the urine, and $32.4 \%$ (range $28.8 \%-35.9 \%$ ) was recovered in the feces. Within 24 hours of dosing, $50.0 \%$ of the dose was recovered in the urine, with over $70 \%$ recovered in both urine and feces within 48 hours and $<1 \%$ recovered in the urine after 3 days postdose.

Based on LC-MS/MS analysis (method A, Supplemental Table 2), the cumulative excreted amount of $(S, R)$-darolutamide was smaller than that of $(S, S)$-darolutamide in urine (Fig. 3B), which reflects the observed ratio of the two diastereomers in plasma. In this analysis, $7.2 \%$ of the administered dose of darolutamide was recovered as darolutamide diastereomers in the urine, consisting of $1.5 \%(S, R)$-darolutamide and $5.7 \%(S, S)$-darolutamide (Fig. 3B). Using LC separation with subsequent off-line radioactivity detection for the investigation of the metabolite profile resulted in a very similar value of $6.7 \%$ excreted as darolutamide in the urine (method B, Supplemental Table 2). The renal clearance of $(S, R)$-darolutamide and $(S, S)$-darolutamide was 1.58 -fold (range 0.93-2.07) and 1.07-fold (range 0.65-1.32) greater, respectively, than the eGFR determined for the individual subjects. The mean ratio of $(S, R)$-darolutamide to $(S, S)$-darolutamide in feces was $1: 7$, determined by an exploratory LC-MS method (method D, Supplemental Table 2), also showing a preference for $(S, S)$-darolutamide.

Metabolite Pattern of Darolutamide in Plasma and Excreta. The profile of darolutamide and its metabolites in plasma obtained by LC with radioactivity detection (method B, Supplemental Table 2 ) is shown in Fig. 4A. Darolutamide and its major metabolite, the oxidation product keto-darolutamide, were the only components quantifiable by LC separation with off-line radioactivity detection in human plasma. Based on $\mathrm{AUC}_{0 \text {-inf }}$ (Table 1), the sum of both compounds in plasma accounted for $87.4 \%$ (28.6\% darolutamide and $58.8 \%$ keto-darolutamide) of total ${ }^{14} \mathrm{C}$-radioactivity [AUC $\mathrm{A}_{0 \text {-inf: }} 159 \mu \mathrm{g}$ _eq. $\mathrm{h} / \mathrm{mL}$ ]; plasma exposure was 2.1-fold higher for keto-darolutamide versus darolutamide. Other metabolites circulating in plasma, i.e., drug glucuronides of darolutamide as well as oxidation products, have only been identified by radioactivity detection at trace levels and did not contribute to the overall exposure to a relevant extent. Detection of nonradiolabeled pyrazole metabolites in the urine (see below), which are likely to be present in plasma prior to urinary excretion, prompted evaluation of plasma for the presence of these metabolites. LC-MS analysis (method C, Supplemental Table 2) detected low levels of the respective nonradiolabeled metabolites M-32, M-33, M-34, and M-36 in plasma.

Radioanalysis for metabolite profiling and identification showed that the urine excretion pattern of darolutamide was more complex than that observed in plasma. The radioactive dose was mostly excreted in urine as $O$-glucuronides (M-7a, M-7b; $25.6 \%$ of dose), $N$-glucuronides (M-15a, M-15b; $5.8 \%$ of dose), and the pyrazole sulfate (M-29; $6.2 \%$ of dose), as well as darolutamide diastereomers (6.7\% of dose, as noted above) (Fig. 4B; Table 2). No relevant amount of keto-darolutamide could be identified in the samples investigated. The absolute structure and stereochemistry of metabolite M-7a and M-15a was confirmed by NMR

TABLE 1

Selected pharmacokinetic parameters of darolutamide, its diastereomers, and keto-darolutamide after a single dose of ${ }^{14} \mathrm{C}$-darolutamide $(300 \mathrm{mg})$ as oral solution Data are presented as geometric means (geometric $\mathrm{CV} \%)(N=6)$.

\begin{tabular}{|c|c|c|c|c|c|}
\hline Plasma PK Parameter & Total ${ }^{14} \mathrm{C}$ Radioactivity & Parent Darolutamide & $(S, R)$-Darolutamide & $(S, S)$-Darolutamide & Keto-Darolutamide \\
\hline $\mathrm{AUC}_{0-\mathrm{inf}}(\mathrm{h} . \mu \mathrm{g} / \mathrm{mL})$ & $159(0.02)^{a}$ & $45.6(0.02)$ & $7.11(0.03)$ & $38.3(0.02)$ & $93.7(0.02)$ \\
\hline$C_{\max }(\mu \mathrm{g} / \mathrm{mL})$ & $16.5(0.02)^{a}$ & $3.82(0.02)$ & $1.81(0.02)$ & $2.49(0.02)$ & $11.7(0.02)$ \\
\hline$T_{\max }(\mathrm{h})^{b}$ & $1.5(1.0-2.0)$ & $1.5(1.0-3.0)$ & $1.0(0.5-1.5)$ & $3.0(1.5-4.0)$ & $1.5(1.0-1.5)$ \\
\hline$t_{1 / 2}(\mathrm{~h})$ & $11.5(24.5)$ & $10.6(22.6)$ & $6.58(20.7)$ & $10.7(20.5)$ & $10.7(31.4)$ \\
\hline Analyte-to-parent ratio ${ }^{c}$ & $\mathrm{NC}$ & $0.185(14.7)^{d}$ & $0.156(12.4)$ & $0.841(2.2)$ & $2.06(11.1)$ \\
\hline
\end{tabular}

NC, not calculated; PK, pharmacokinetic; $T_{\max }$, time when maximum plasma concentration is observed.

${ }^{a}$ Units are $\mu \mathrm{g}$ eq of ${ }^{14} \mathrm{C}$-darolutamide.

${ }^{b}$ Median (range).

${ }^{c}$ Based on individual $\mathrm{AUC}_{0 \text {-inf. }}$.

${ }^{d}$ Diastereomer ratio, calculated as $(S, R)$-darolutamide/( $\left.S, S\right)$-darolutamide. 
A

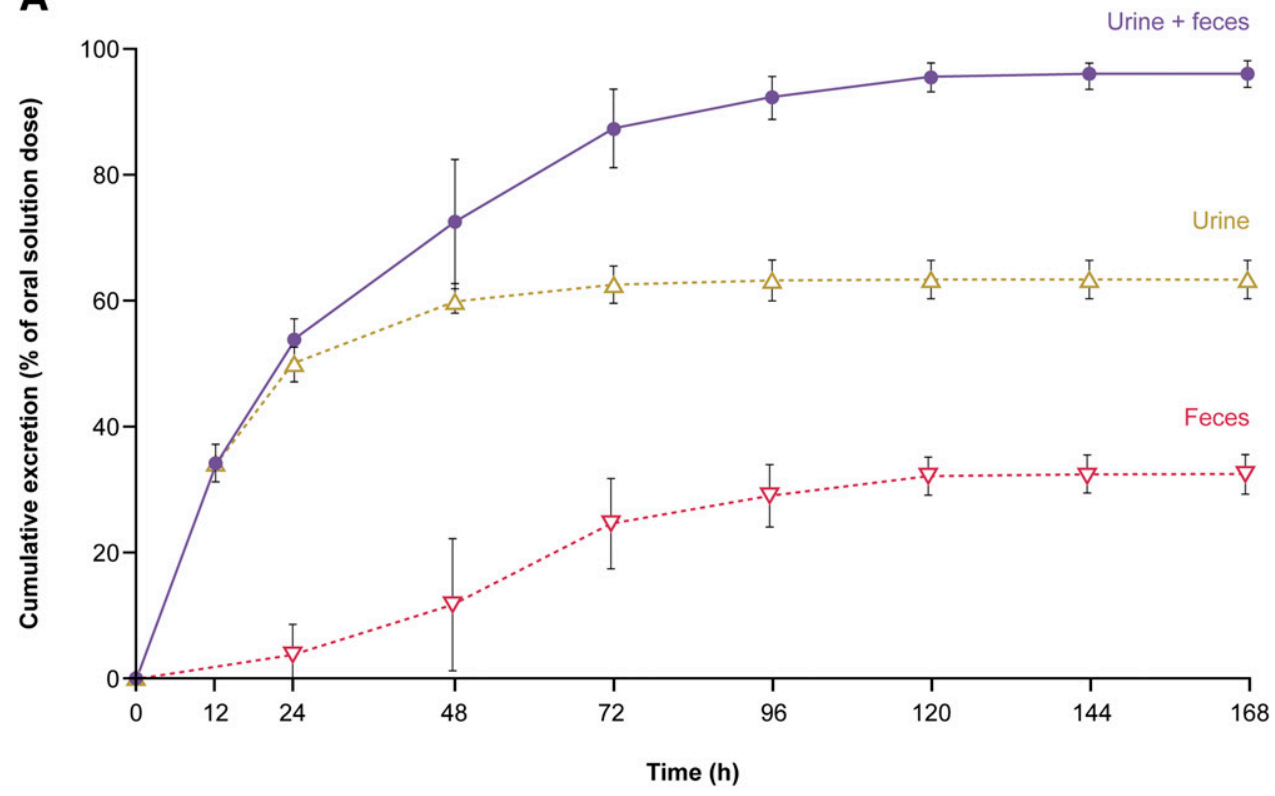

B

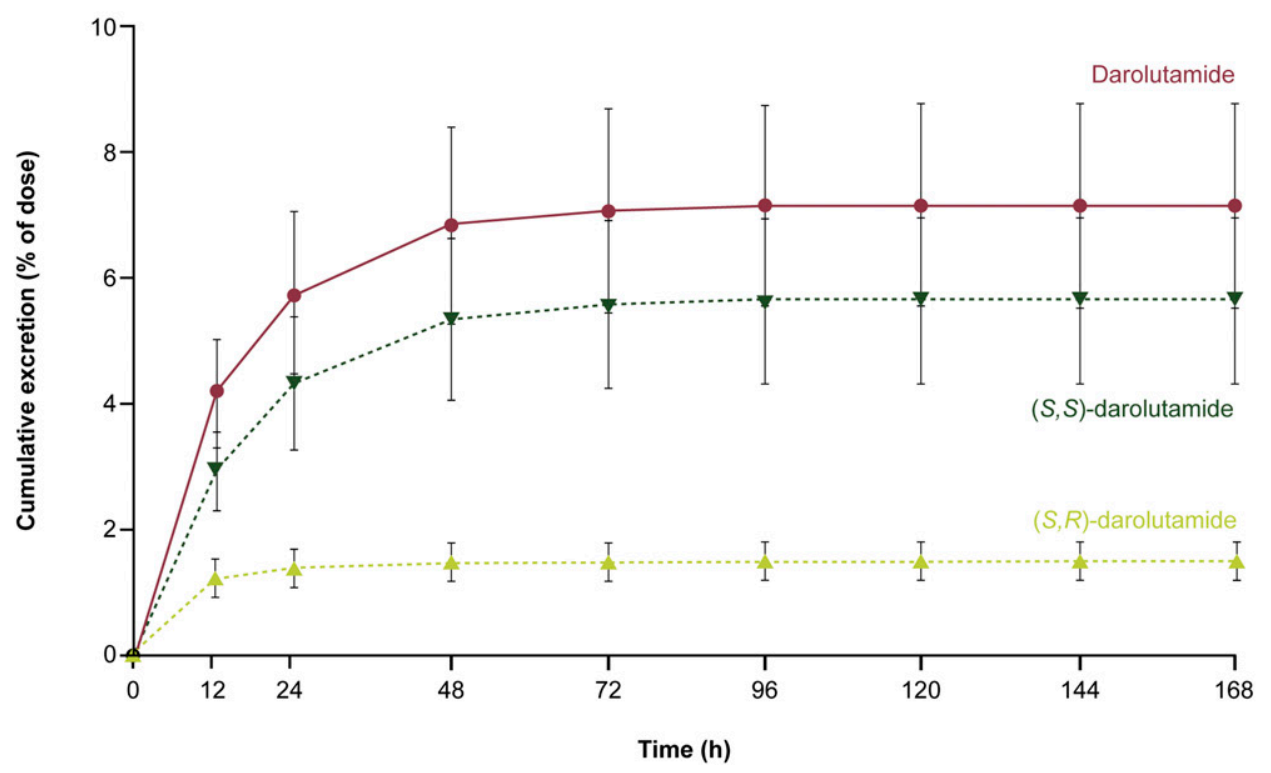

Fig. 3. Cumulative (A) excretion of total radioactivity in urine and feces and (B) excretion and recovery of darolutamide and its diastereomers in urine after a single dose of ${ }^{14} \mathrm{C}$-darolutamide $(300 \mathrm{mg})$ as oral solution. Data are presented as arithmetic means for cumulative percentage of amounts excreted $(n=6)$. with material isolated from urine (Supplemental Table 5). This material was further used to chromatographically assign and differentiate between the $O$ - and $N$-glucuronide diastereomers $\mathrm{M}-7 \mathrm{a} / \mathrm{M}-7 \mathrm{~b}$ and $\mathrm{M}$ 15a/M-15b. Using LC high-resolution mass spectrometry (method C, Supplemental Table 2), metabolites that lost the ${ }^{14} \mathrm{C}$-label because of cleavage of darolutamide and/or keto-darolutamide were identified and evaluated in urine: these structural counterparts to metabolites as M-21, M-22, M-24, and M-29 accounted for $3.2 \%, 3.8 \%, 4.2 \%$, and $6.2 \%$ of dose, respectively. Overall, $94.0 \%$ of the radioactivity present in urine samples (59.6\% of the dose) could be assigned to known structures.

The ratios of $O$-glucuronides $\mathrm{M}-7 \mathrm{a}$ to $\mathrm{M}-7 \mathrm{~b}$ and $\mathrm{N}$-glucuronides M-15a to M-15b, identified using an LC-MS method enabling chiral separation (method E, Supplemental Table 2), increased over time in all subjects. Noting that "a"-suffixed glucuronides originate from $(S, S)$ darolutamide and " $\mathrm{b}$ "-suffixed glucuronides originate from $(S, R)$-darolutamide, there was an overall preference for formation of the $(S, S)$ - over the $(S, R)$ diastereomer, with 14-fold (M-7a/M-7b) and 8-fold (M-15a/M-15b) greater amounts formed, respectively (Table 2 ).

The radioactivity found in fecal extracts was mainly assigned as darolutamide diastereomers, keto-darolutamide (M-1), and carboxylic acid (M-28, formed by oxidation of keto-darolutamide), with $30.3 \%$, $0.6 \%$ and $1.0 \%$ of dose, respectively (Fig. 4C; Table 2). Overall, $98.4 \%$ of the radioactivity present in fecal samples (31.9\% of dose) could be attributed to known structures. When the stability of ketodarolutamide was investigated in human feces samples under anaerobic conditions, results indicated that keto-darolutamide was prone to reduction to darolutamide in the environment of the gastrointestinal tract (Supplemental Fig. 1).

Overall, the metabolite profiles of darolutamide in urine and feces revealed darolutamide diastereomers, $\mathrm{O}$ - and $\mathrm{N}$-glucuronides of the 

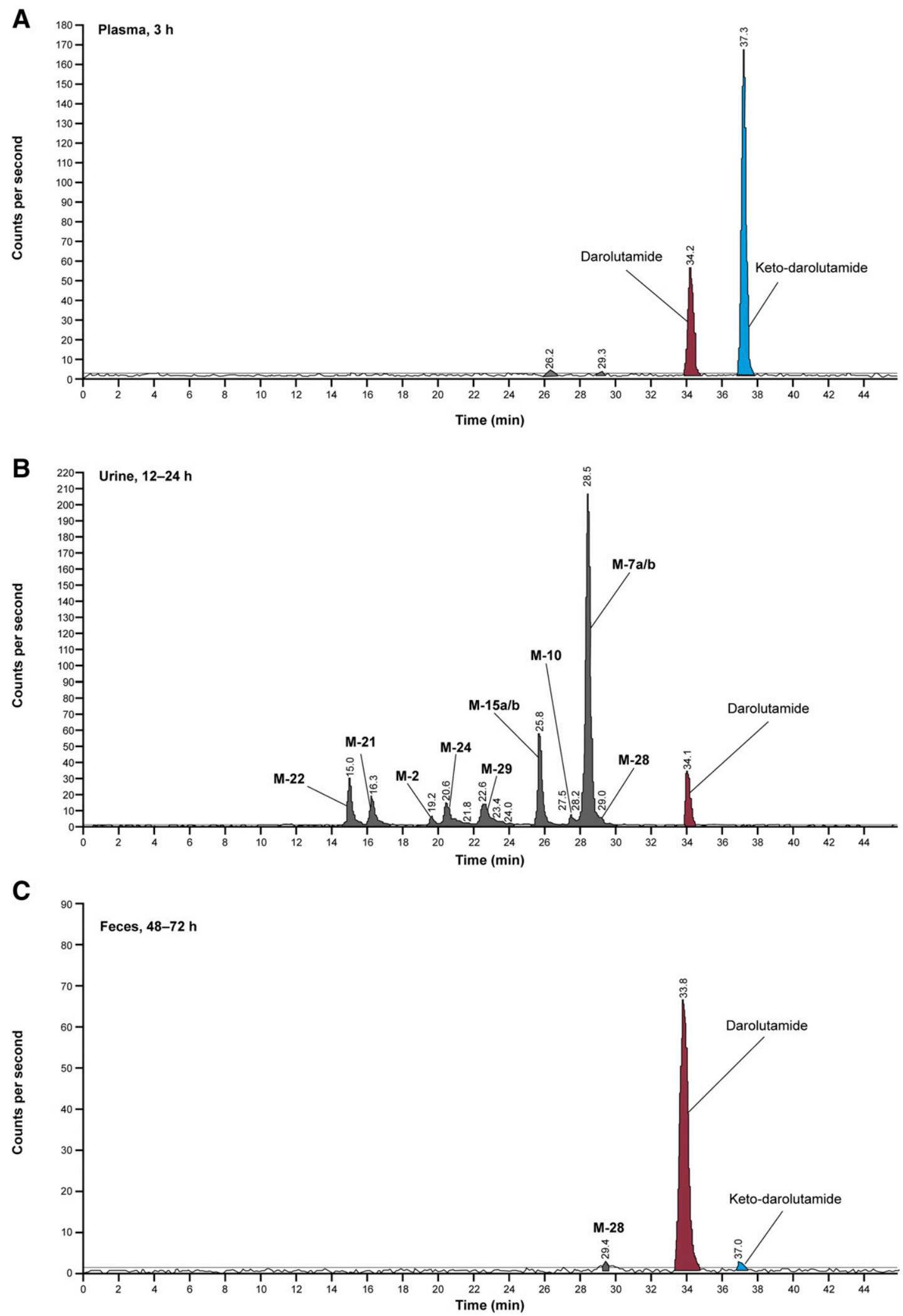

Fig. 4. ${ }^{14} \mathrm{C}$-Darolutamide metabolic profiles in (A) plasma collected at 3 hours postdose, (B) urine collected from 12 to 24 hours postdose, and (C) feces collected from 48 to 72 hours postdose from an individual participant after oral administration of $300 \mathrm{mg}$ darolutamide containing $5.4 \mathrm{MBq}{ }^{14} \mathrm{C}$-darolutamide (obtained using nonchiral LC with off-line radioactivity detection). 
TABLE 2

Metabolite profile of darolutamide in excreta, expressed as percentage of the administered dose, as found in urine and feces $0-168 \mathrm{~h}$ after administration of ${ }^{14} \mathrm{C}$-darolutamide $(300 \mathrm{mg})$ as oral solution

Data are presented as arithmetic means.

\begin{tabular}{|c|c|c|c|}
\hline \multirow{2}{*}{ Component } & \multirow{2}{*}{ Type } & \multicolumn{2}{|c|}{$\operatorname{Dose}^{a}$} \\
\hline & & Urine & Feces \\
\hline & & \multicolumn{2}{|c|}{$\%$} \\
\hline M-1 & Oxidation product (keto-darolutamide) & ${ }^{b}$ & 0.6 \\
\hline M-2 & Hydroxylated and glucuronidated metabolite & 1.3 & $c^{c}$ \\
\hline M-7a & $O$-glucuronide & 23.8 & $-^{c}$ \\
\hline M-7b & $O$-glucuronide & 1.8 & $-^{c}$ \\
\hline M-10 & Glucuronidation product of M-1 & 0.6 & ${ }^{c}$ \\
\hline M-15a & $N$-glucuronide & 5.0 & $-^{c}$ \\
\hline$M-15 b$ & $N$-glucuronide & 0.7 & $-^{c}$ \\
\hline $\mathrm{M}-21$ & Dealkylated product of oxidative cleavage & 3.2 & $-^{c}$ \\
\hline M-22 & Dealkylated product of oxidative cleavage & 3.8 & ${ }^{c}$ \\
\hline M-24 & Dealkylated product of oxidative cleavage & 4.2 & ${ }^{c}$ \\
\hline M-28 & Oxidation product of M-1 & 2.4 & 1.0 \\
\hline M-29 & Dealkylated product of oxidative cleavage & 6.2 & $-^{c}$ \\
\hline Darolutamide & & 6.7 & 30.3 \\
\hline Other & & 3.8 & 0.5 \\
\hline$\% \mathrm{~A}_{\mathrm{e}}(\%$ of dose $)$ & & 63.4 & 32.4 \\
\hline
\end{tabular}

${ }^{a}$ Percentage of oral dose.

${ }^{b}$ - not found in urine.

${ }_{-}$, not found in feces.

diastereomers (M-7 and M-15), and several phase I biotransformation products resulting from cleavage of the drug molecule into separate moieties, with the labeled metabolites M-21, M-22, M-24, and M-29 on one side and the corresponding unlabeled metabolites M-32, M-33, M34, and M-36 on the other side. Molecular and key fragment ions of darolutamide and metabolites are presented in Supplemental Table 6. A schematic representation of the proposed metabolic pathway is depicted in Fig. 5.

\section{In Vitro Studies}

Metabolite Profile in Human Hepatocytes. After an incubation of ${ }^{14} \mathrm{C}$-darolutamide with human hepatocytes, the most abundant metabolites were keto-darolutamide and $O$-glucuronides (M-7 isomers; analysis using method G, Supplemental Table 4). Only minor formation of other metabolites was found (Table 3). Applying itraconazole as a CYP3A4 inhibitor in hepatocytes, the metabolic clearance of darolutamide was reduced by approximately $30 \%$ (Supplemental Table 7), confirming involvement of CYP3A4.

Oxidative and Reductive Metabolism and Interconversion of Diastereomers of Darolutamide. Oxidative and reductive metabolism of darolutamide and the mechanisms of the interconversion of the two diastereomers were investigated in various in vitro assays (analyzed with LC-MS method I and J, Supplemental Table 4).

The oxidation of darolutamide was studied by incubation of ${ }^{14} \mathrm{C}$-darolutamide in HLM as well as a panel of 21 recombinant human P450 isoforms. CYP3A4 and, to a lesser extent, CYP1A1 were the main enzymes catalyzing the depletion of ${ }^{14} \mathrm{C}$-darolutamide, with predominant formation of keto-darolutamide (Fig. 6). In addition, formation of the metabolites M-26, M-30, and M-31 by further oxidation and cleavage of the molecule was observed (Fig. 5). Oxidative formation of these metabolites was also mediated via CYP3A4 and to a very minor extent via other recombinant P450 isoforms. The role of CYP3A4 in the formation of keto-darolutamide was confirmed using CYP3A4-specific inhibitors (ketoconazole, cobicistat) in microsomal incubations.

Minor amounts of keto-darolutamide were also observed in HLM and HLC incubations of darolutamide in the presence of the cofactors NAD and NADP. Despite the very slow formation rate compared with P450mediated metabolism, these results suggest that other cytosolic and membrane-associated enzymes can also form keto-darolutamide (Fig. 7). After applying different assay conditions, the results suggest that alcohol dehydrogenase ( $\mathrm{pH}$ - and NAD-dependent metabolism) and AKRs (NADP-dependent metabolism) are able to form minor amounts of keto-darolutamide from darolutamide. Notably, when incubating the diastereomers individually, the $(S, S)$-diastereomer appears to be the preferred substrate for these non-P450 oxidation pathways (Supplemental Fig. 2).

The oxidation and reduction of keto-darolutamide was studied in HLM, HLC, and several recombinant enzymes. The reduction back to darolutamide was efficient in HLC in the presence of reduced NADP (NADPH), and it was sensitive to the AKR inhibitors flufenamic acid (AKR1C1-4) and EM1404 (AKR1C3) (Fig. 8). Diastereomer-specific analysis suggested that $(S, S)$-darolutamide is specifically produced by cytosolic enzymes, whereas both diastereomers were detected in incubations of HLM and hepatocytes (Fig. 9). Recombinant AKR1C1-1C4 incubations confirmed the dominant role of AKR1C3 in reductive metabolism (Fig. 8). Furthermore, AKR1D1 was observed to have some activity (data not shown). HLM incubation using ketodarolutamide as substrate revealed both reduction and oxidation (M-30, M-31, M-26, M25; Fig. 5). Several P450 isoforms are able to metabolize keto-darolutamide, as shown in incubations applying recombinant enzymes (Supplemental Fig. 3).

The interconversion of diastereomers was studied in human hepatocytes using diastereomer-specific LC-MS analytics (method I, Supplemental Table 4) after incubation of the substrates $(S, R)$-darolutamide, $(S, S)$-darolutamide, and keto-darolutamide in the presence and absence of AKR1C3 inhibitor BAY 1128688 or CYP3A4 inhibitor cobicistat. In the presence of cobicistat, depletion of $(S, R)$-darolutamide and $(S, S)$-darolutamide was less pronounced compared with the presence of no inhibitor (Fig. 10A), indicating the involvement of CYP3A4 in the metabolism of both diastereomers. The depletion of keto-darolutamide was not significantly impacted by cobicistat, whereas the addition of BAY 1128688 resulted in a distinctly slower depletion of keto-darolutamide (Fig. 10A), indicating that reduction via AKR1C3 plays the major role in the metabolism of keto-darolutamide. In the presence of BAY 1128688 , there was minor inhibition of $(S, R)$ darolutamide depletion, and further depletion of $(S, S)$-darolutamide 


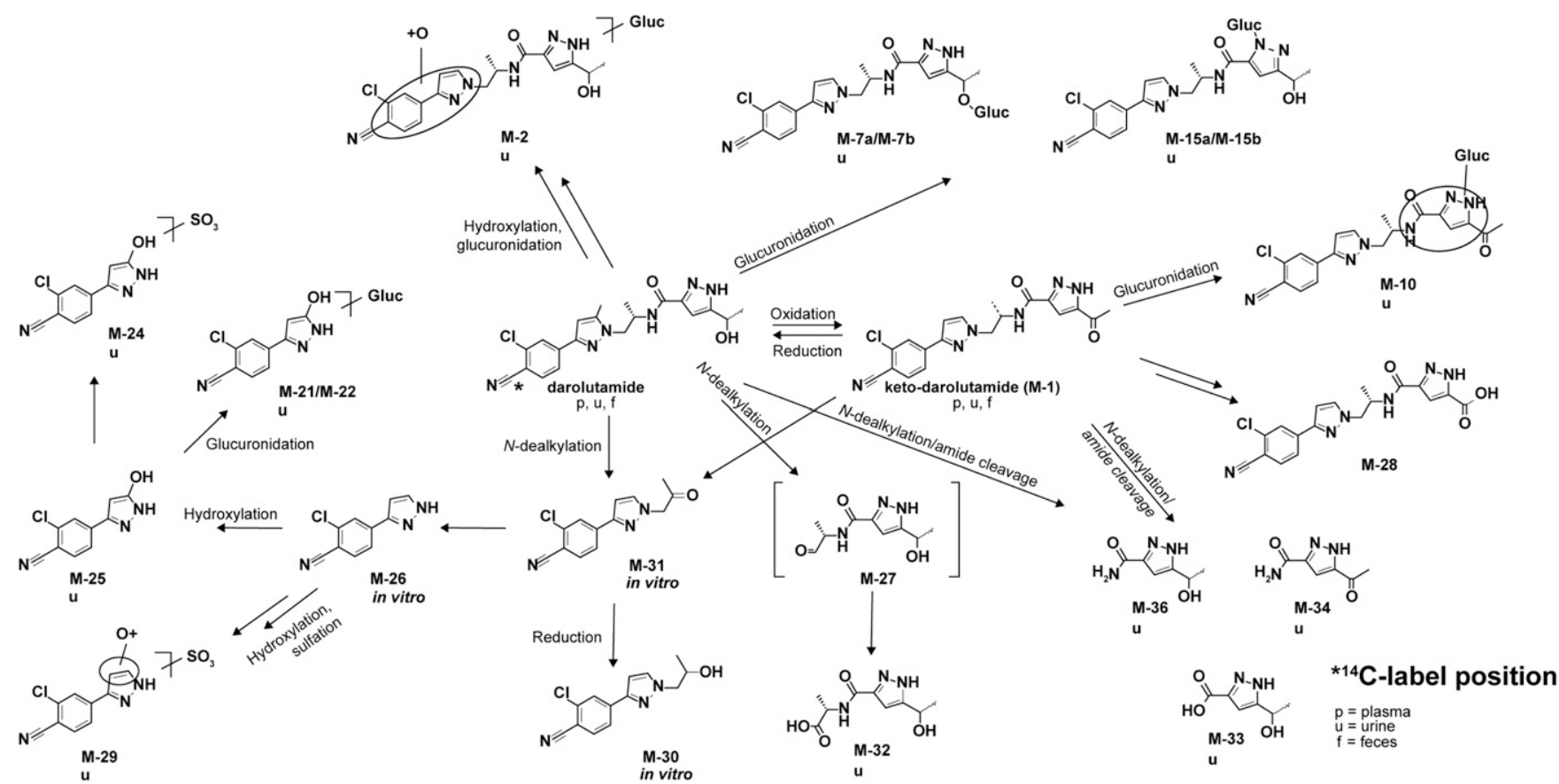

Fig. 5. Proposed metabolic pathways for ${ }^{14} \mathrm{C}$-darolutamide in humans. f, feces; Gluc, glucuronide; p, plasma; u, urine.

occurred. Formation of the respective metabolites in this interconversion process is summarized in Fig. 10B. Applying $(S, R)$-darolutamide as starting material, clear time-dependent formation of keto-darolutamide as well as $(S, S)$-darolutamide was observed, whereas the formation of $(S, R)$-darolutamide from starting material $(S, S)$-darolutamide was much lower. This was confirmed when keto-darolutamide was used as a substrate. A rapid increase in $(S, S)$-darolutamide was observed, reaching maximum concentration after 30 minutes of incubation, with very low simultaneous formation of $(S, R)$-darolutamide. This observation indicates preferred formation of $(S, S)$-darolutamide in hepatocytes.

Thus, the oxidative metabolism in hepatocytes of both darolutamide diastereomers leading to formation of keto-darolutamide is mediated by CYP3A4, AKR1C3 stereoselectively catalyzes the reduction of ketodarolutamide to $(S, S)$-darolutamide, and other reductases contribute to formation of $(S, R)$-darolutamide.

Glucuronidation. In experiments with recombinantly expressed UGTs, glucuronidation of darolutamide to yield the diastereomeric $O$-glucuronides $\mathrm{M}-7 \mathrm{a}$ and $\mathrm{M}-7 \mathrm{~b}$ was predominantly catalyzed by UGT1A9 and, to a lesser extent, by UGT1A8, UGT2B17, UGT1A7, UGT1A3, and UGT1A1 (Supplemental Fig. 4). Formation of the diastereomeric $N$-glucuronides M-15a and M-15b was mediated solely by UGT2B10. Experiments in which the single diastereomers were investigated separately were in good agreement with those performed with darolutamide.

Greater amounts of the drug glucuronides were formed in liver microsomes than in either renal or intestinal microsomes, and the metabolite pattern differed among the tested tissues (Fig. 11; analyzed with method $\mathrm{H}$, Supplemental Table 4). In liver microsomes, the $\mathrm{O}$-glucuronide $\mathrm{M}$-7a and $\mathrm{N}$-glucuronides $\mathrm{M}$-15a [glucuronides of $(S, S)$-darolutamide] were formed in an approximately 1:1 ratio, and their formation was more pronounced than that of M-7b and M-15b [glucuronides of $(S, R)$-darolutamide]. Renal and intestinal microsomes showed a high preference for the formation of M-7a over M-7b, and levels of M-15a and M-15b were very low because of the absence of UGT2B10 in these tissues.
In vitro experiments using HLM in the presence of UGT isoformselective inhibitors further evaluated the relative contribution of single UGT isoforms to darolutamide glucuronidation. Partial inhibition of M7a and M-7b formation by atazanavir (a UGT1A1 and UGT1A3 inhibitor; $10 \mu \mathrm{M}$ ) and niflumic acid (a UGT1A9 inhibitor; $10 \mu \mathrm{M}$ ) indicated that UGT1A9, UGT1A1, and UGT1A3 are predominantly responsible for $O$-glucuronidation of darolutamide. The combination of both inhibitors reduced M-7a and M-7b formation by approximately $80 \%$ and $75 \%$, respectively. In the presence of the UGT2B10-selective inhibitor desloratadine, formation of $\mathrm{M}-15 \mathrm{a}$ and $\mathrm{M}-15 \mathrm{~b}$ was reduced versus control by $79 \%$ and $62 \%$, respectively, confirming that UGT2B 10 is the major isoform mediating $N$-glucuronidation. A correlation analysis of experiments using HLM from individual donors (see Supplemental Table 8) confirmed the involvement of UGT2B10 in M-15a and M-15b formation $\left(r^{2}=0.83\right.$ and $r^{2}=0.84$, respectively; substrate: levomedetomidine).

To further evaluate the relative contribution of UGT1A1 and UGT1A9 to the glucuronidation of darolutamide, RAFs in HLM were determined for both diastereomers of darolutamide (see Supplemental Table 9). The relative contributions of UGT1A1 and UGT1A9 to $(S, R)$ -

TABLE 3

Metabolite profiles in incubations of ${ }^{14} \mathrm{C}$-darolutamide $(5 \mu \mathrm{M})$ with human hepatocytes from three donors (suspension incubation for $3 \mathrm{~h}, 1$ million cells $/ \mathrm{mL}$, selected metabolites)

\begin{tabular}{lc}
\hline & $\begin{array}{c}\text { Mean of Total } \\
\text { Radioactivity }\end{array}$ \\
\hline Darolutamide & $\%$ \\
Keto-darolutamide & 70.7 \\
M-7 & 21.5 \\
M-4 & 3.7 \\
Others $^{b}$ & 2.2 \\
\hline
\end{tabular}

${ }^{a}$ Coelution with two additional hydroxylated metabolites (M-6, M-5).

${ }^{b}$ Comprises M-2, M-3, M-8, and other unidentified compounds. 


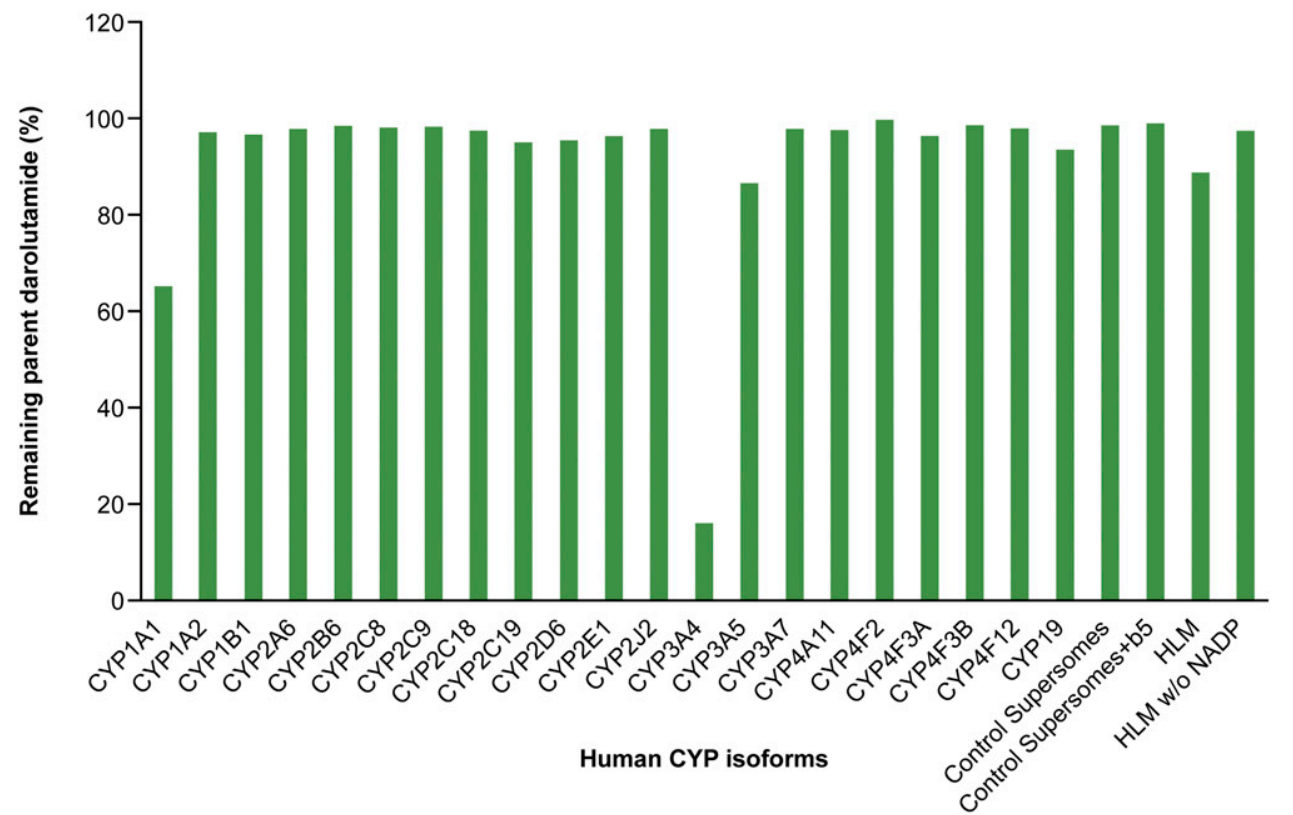

Fig. 6. Depletion of ${ }^{14} \mathrm{C}$-darolutamide $(1 \mu \mathrm{M})$ after 1-hour incubation with a complete panel of all available recombinant P450 enzymes $(100 \mathrm{pmol} / \mathrm{mL})$ or human liver microsomes $(0.5 \mathrm{mg} / \mathrm{mL})$. CYP, cytochrome P450; w/o, without.

and $(S, S)$-darolutamide glucuronidation were in good accordance with the values calculated based on chemical inhibition experiments.

\section{Discussion}

The presented phase I study in which healthy male subjects received $300 \mathrm{mg}{ }^{14} \mathrm{C}$-darolutamide as an oral solution, supported by in vitro data also presented here, together provide extensive information about the pharmacokinetics, biotransformation, and excretion of darolutamide and its metabolites.

After oral solution administration, darolutamide was rapidly absorbed. Parent drug and keto-darolutamide accounted for over $87 \%$ of total ${ }^{14} \mathrm{C}$-radioactivity in plasma, with keto-darolutamide contributing the majority. The equal ratio of the two diastereomers in the administered dose changed to a 1:5 ratio favoring $(S, S)$-darolutamide in plasma. In vitro studies in human hepatocytes showed that this change is due to an enzymatic interconversion process: the conversion of $(S, R)$-darolutamide to $(S, S)$-darolutamide was rapid and distinct, whereas the conversion from
$(S, S)$-darolutamide to $(S, R)$-darolutamide was less pronounced. Furthermore, the $(S, R)$-diastereomer was found to have a shorter half-life in vivo than the $(S, S)$-diastereomer. We have previously shown in rats in vivo that, after dosing of one individual diastereomer, the other diastereomer also appears, with a slight preference for $(S, S)$-darolutamide formation after repeated dosing (Taavitsainen et al., 2020). The diastereomer interconversion takes place in each species studied (including mice, rats, dogs, and humans), with the prevailing diastereomer differing between species (Nykänen et al., 2020; Taavitsainen et al., 2020) (data on file). However, in all species, all three compounds (the diastereomers and the ketone metabolite) are found in plasma, but at different ratios. All three substances show similar pharmacological activity (Moilanen et al., 2015).

Excretion of darolutamide and its biotransformation products is rapid and complete. Approximately two-thirds of the administered dose was excreted via the kidneys in urine. Only small amounts of approximately $7 \%$ of the administered dose were excreted as the two diastereomers in

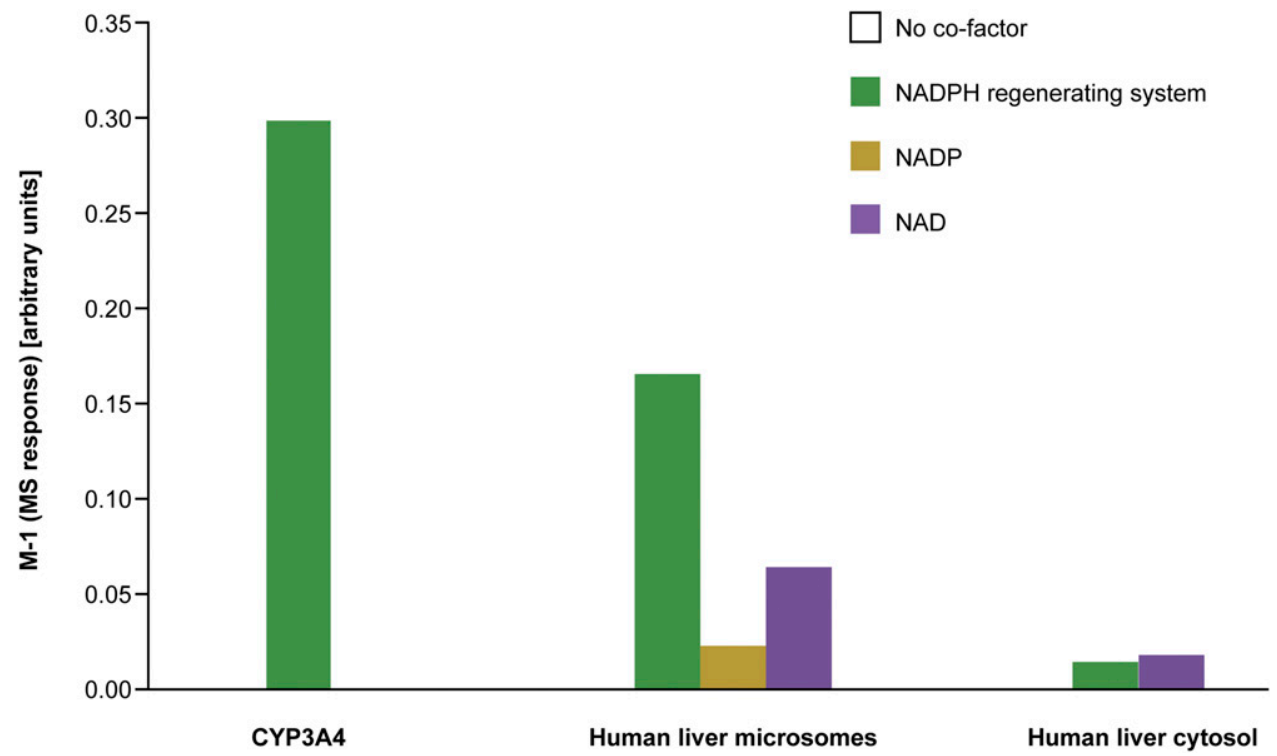

Fig. 7. Formation of keto-darolutamide (M1) from darolutamide $(1 \mu \mathrm{M})$ in cell-free reaction with recombinant CYP3A4 (50 pmol/mL), human liver microsomes $(0.5 \mathrm{mg} / \mathrm{mL})$, and human liver cytosol $(0.5 \mathrm{mg} / \mathrm{mL})$ after 60 minutes of incubation, dependent on cofactor supplementation. MS, mass spectrometry. 


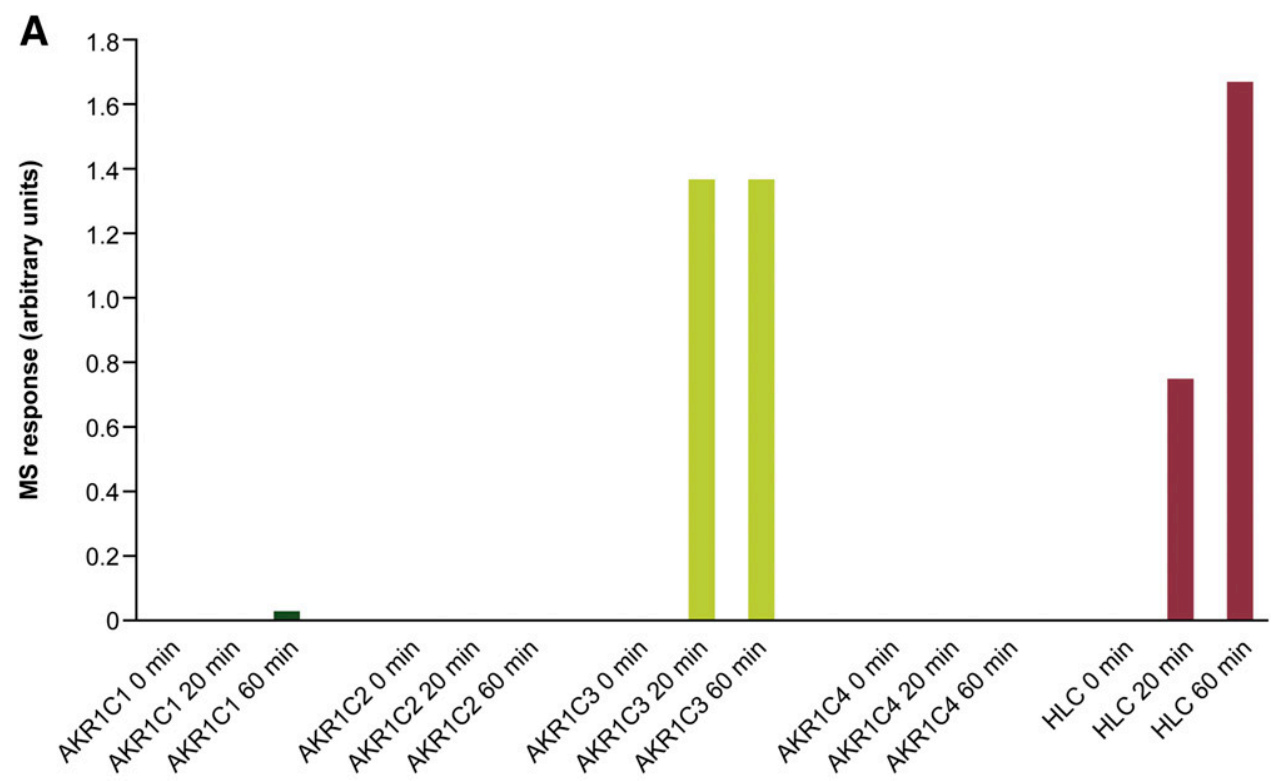

B

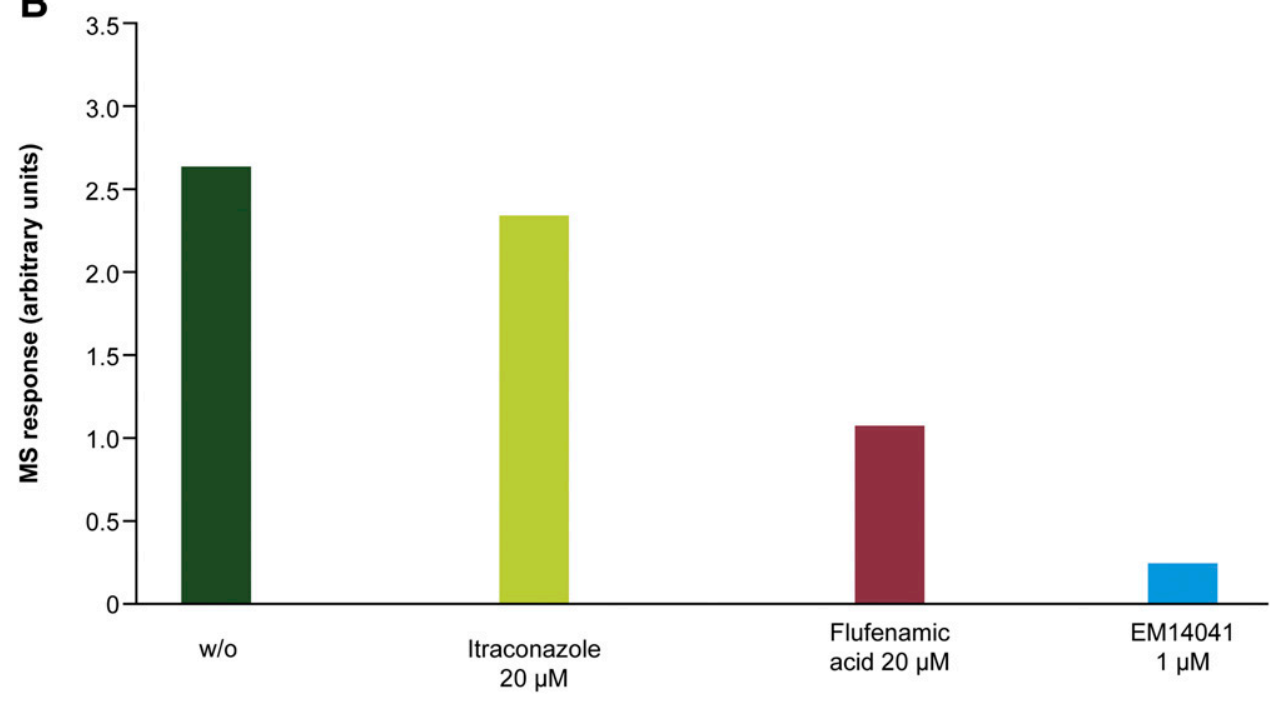

Fig. 8. Reduction of keto-darolutamide $(1 \mu \mathrm{M})$ to darolutamide after incubation $(0,20,60$ minutes) in recombinant human $(50 \mathrm{nM})$ AKR1C1, AKR1C2, AKR1C3, AKR1C4, and human liver cytosol $(0.5 \mathrm{mg} / \mathrm{mL})(\mathrm{A})$ and the effect of chemical inhibitors on formation of darolutamide from keto-darolutamide $(1 \mu \mathrm{M})$ after 60-minute incubation in human liver cytosol $(0.5 \mathrm{mg} / \mathrm{mL})$ (B). Flufenamic acid (inhibitor of AKR1C1-1C4) and EM1404 (synthesized at Bayer AG, Germany, AKR1C3 inhibitor) limited formation of darolutamide. Itraconazole is a known CYP3A4 inhibitor. MS, mass spectrometry; w/o, without inhibitor. urine, demonstrating extensive metabolism of darolutamide. The renal clearance of the diastereomers was similar to the estimated eGFR with a tendency for a slightly higher renal clearance of $(S, R)$-darolutamide. The data indicate that darolutamide is mainly excreted by passive glomerular filtration, although a contribution of transporter-mediated secretion cannot be excluded in the case of $(S, R)$-darolutamide. In line with the 1:5 plasma ratio of $(S, R)$-darolutamide to $(S, S)$-darolutamide, the excreted amount of $(S, R)$-darolutamide in urine was smaller than that of $(S, S)$-darolutamide, with an observed ratio of 1:4.

Excretion of the radioactive dose into urine occurred mainly as $O$-glucuronides of the darolutamide diastereomers, with smaller amounts as $N$-glucuronides, pyrazole sulfate, and darolutamide. In addition, nonlabeled pyrazole amide was measured at a relatively high proportion in urine. Metabolite profiling in urine indicated a preference for $(S, S)$ - over $(S, R)$-darolutamide to form the respective $O$-glucuronides, as the difference in urine concentrations of M-7a and M-7b was almost 14-fold. In vitro investigations in HLM provided further support for more pronounced $O$-glucuronidation of $(S, S)$-darolutamide versus $(S, R)$-darolutamide.
Although the majority of drug-related radioactivity was excreted renally, the high degree of metabolite formation suggests that hepatic metabolism contributes to darolutamide clearance more than renal elimination.

Approximately one-third of the administered dose was excreted with feces. Darolutamide was the predominant component in fecal extracts. Keto-darolutamide and, to a lesser extent, the carboxylic acid metabolite of darolutamide were identified as the most relevant metabolites but amounted to less than $2 \%$ of the dose. Excretion of drug diastereomers in the feces could result via three different clearance scenarios: both drug diastereomers are excreted directly into feces via biliary transportermediated pathways [darolutamide has been identified as a substrate for P-glycoprotein (Zurth et al., 2019)], the observed instability of ketodarolutamide in the reducing anaerobic microbiome in the human gastrointestinal tract could account for the high proportion of darolutamide, or secreted drug glucuronides are cleaved in the presence of the microbial gut flora back to the respective drug isomers. Biliary excretion has been studied in rats dosed with ${ }^{14} \mathrm{C}$-darolutamide (Taavitsainen et al., 2020). The rat metabolite profile in bile supports direct biliary 


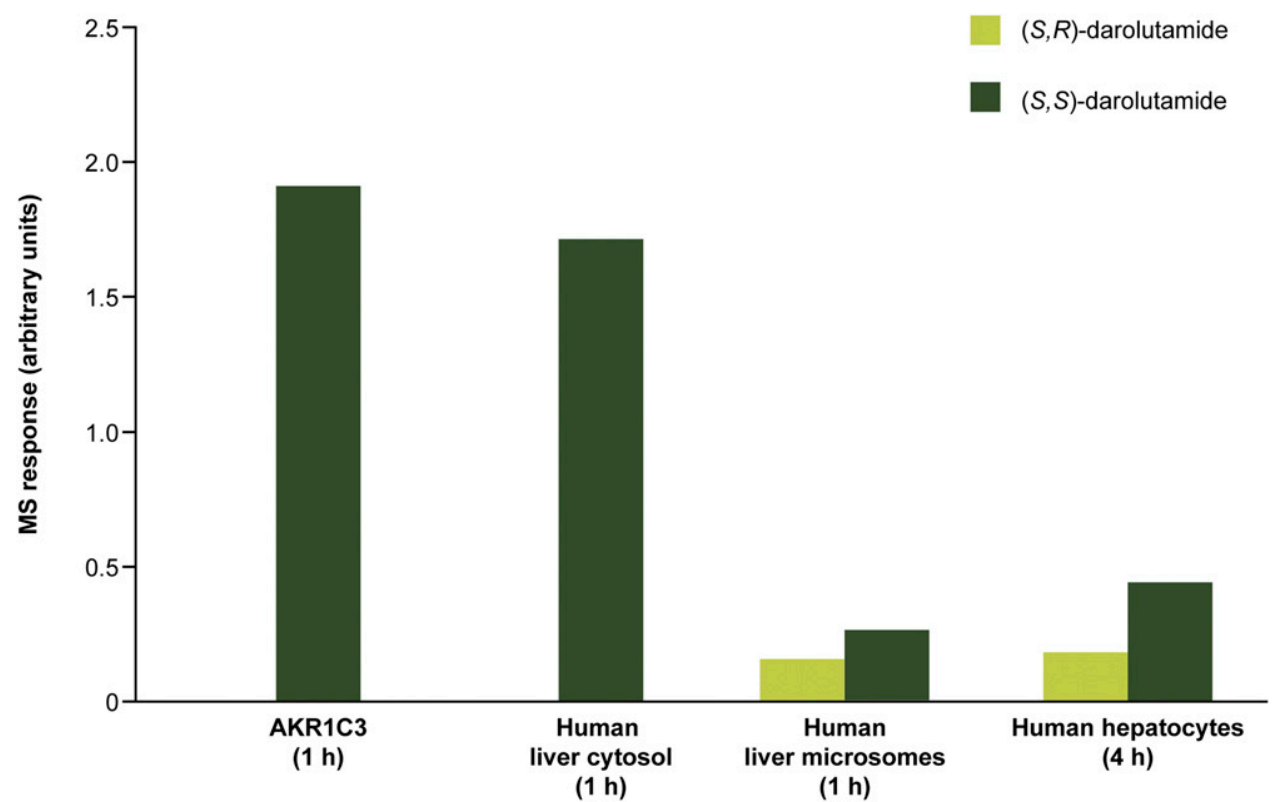

Fig. 9. Formation of diastereomers from ketodarolutamide $(1 \mu \mathrm{M})$ in vitro with recombinant AKR1C3 $(50 \mathrm{nM})$, human liver cytosol $(0.5 \mathrm{mg} / \mathrm{mL})$, human liver microsomes $(0.5 \mathrm{mg} / \mathrm{mL})$, and human hepatocytes $\left(10^{6}\right.$ cells $\left./ \mathrm{mL}\right)$. MS, mass spectrometry. excretion of darolutamide diastereomers (approximately $8 \%$ of dose) as well as excretion of $O$-glucuronide conjugates (approximately $6 \%$ of dose). However, at least in the rat, the proportion of ketodarolutamide in bile was very small, being only approximately $0.1 \%$ of the dose (Taavitsainen et al., 2020). Differences in the glucuronidation of $(S, S)$-darolutamide and $(S, R)$-darolutamide may contribute to the 1:7 ratio of $(S, R)$ - versus $(S, S)$-darolutamide seen in feces, a more pronounced difference than that seen in plasma and urine. The slightly higher proportion of $(S, S)$-darolutamide in fecal extracts compared with plasma may be due to breakdown of the
$O$-glucuronides by $\beta$-glucuronidases. Based on the studies presented, the actual in vivo situation for excretion of the diastereomers may result from a combination of all three of the proposed scenarios. However, in patients with nmCRPC receiving darolutamide in tablet formulation with food, for which absorption is 60\%-70\% (Massard et al., 2016) (in contrast to the complete absorption of the oral solution used in this study), unabsorbed drug can also contribute to the amount of darolutamide excreted in feces.

Further characterization of the metabolic profile of darolutamide in vitro indicated that the interconversion of the two diastereomers is

$$
\begin{array}{lll}
\rightarrow-\text { No inhibitor } & \checkmark \text { No inhibitor } & \rightarrow \text { No inhibitor } \\
\rightarrow-\text { BAY1128688 } & \checkmark \text { BAY1128688 } & \rightarrow \text { BAY1128688 } \\
\rightarrow-\text { Cobicistat } & \checkmark \text { Cobicistat } & \rightarrow \text { Cobicistat }
\end{array}
$$

A
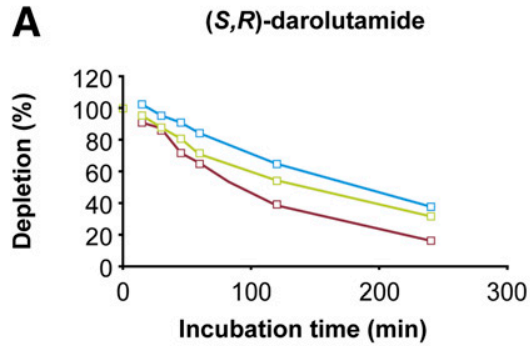

B

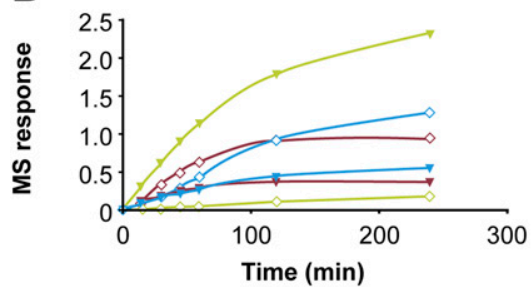

$(\mathrm{S}, \mathrm{S})$-darolutamide
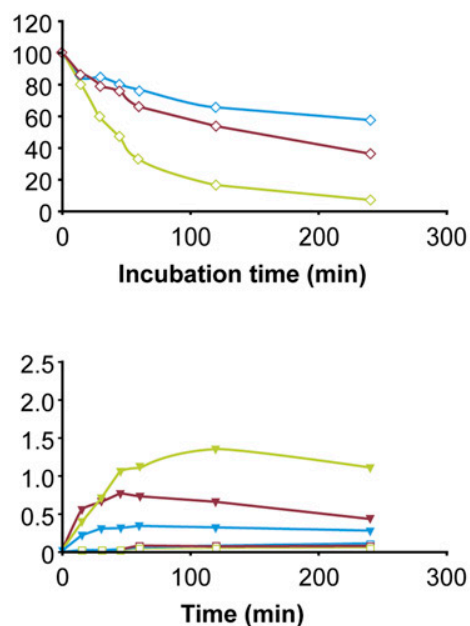

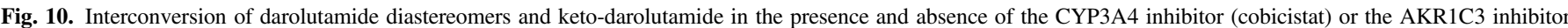

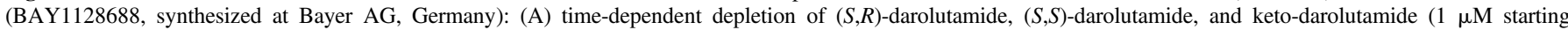

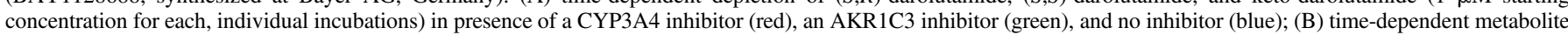

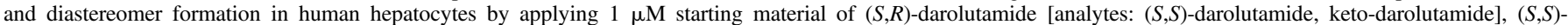

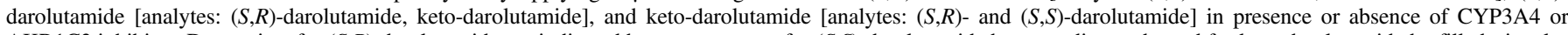

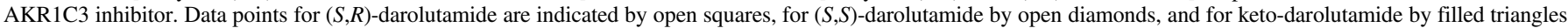
throughout. MS, mass spectrometry. 


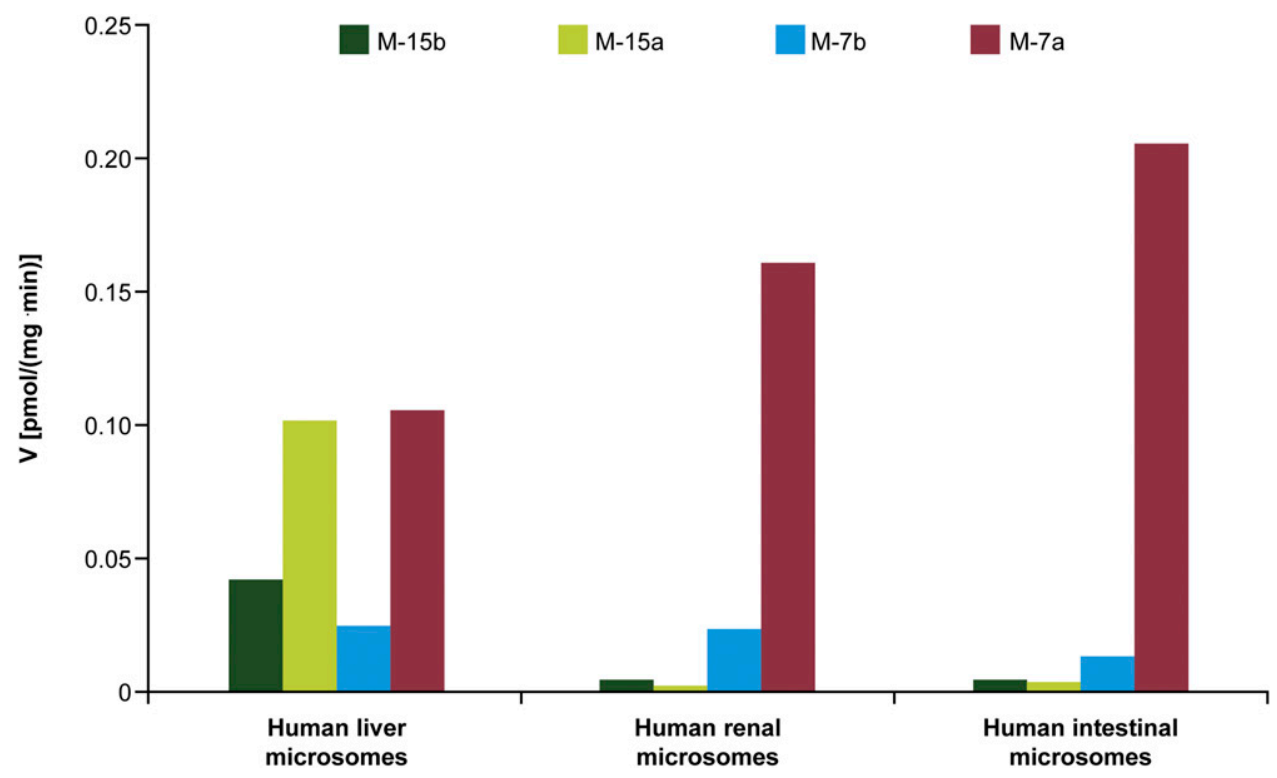

Fig. 11. Formation of glucuronides from darolutamide $(1 \mu \mathrm{M})$ in human liver $(0.5 \mathrm{mg} /$ $\mathrm{mL})$, renal, and intestinal microsomes $(0.5 \mathrm{mg} /$ $\mathrm{mL}$ ) after 1 hour, identified using an LC-MS method enabling separation of the diastereomers. M-7a and $\mathrm{M}-7 \mathrm{~b}$ are $O$-glucuronides of $(S, S)$-darolutamide and $(S, R)$-darolutamide, respectively. M-15a and $\mathrm{M}-15 \mathrm{~b}$ are $\mathrm{N}$-glucuronides of $(S, S)$-darolutamide and $(S, R)$-darolutamide, respectively. $\mathrm{V}$, reaction velocity. a two-step process starting with oxidation to keto-darolutamide followed by reduction. This process is catalyzed by an interplay of membraneassociated enzymes (mainly CYP3A4, minor CYP1A1) as well as cytosolic enzymes. The role of CYP3A4 in the oxidative metabolism of darolutamide was supported by phase I drug-drug interaction studies (Zurth et al., 2019). In addition, as a P450-independent pathway, alcohol dehydrogenase also contributes to oxidation of darolutamide to ketodarolutamide to a minor extent. Cytosolic NADP-dependent AKR activity also made a minor contribution to the oxidative pathway, forming keto-darolutamide from $(S, S)$-darolutamide as the preferred substrate. In hepatocytes, keto-darolutamide is very rapidly reconverted to darolutamide with a preferred formation of $(S, S)$-darolutamide. This reduction is predominantly catalyzed by cytosolic reductase(s), with AKR1C3 playing the major role. Furthermore, other reductases present in cytosol along with microsomal reductases may also contribute to this biotransformation reaction to a minor degree. The stereoselectivity of cytosolic AKR1C3 results in the preferential formation of $(S, S)$ darolutamide from keto-darolutamide, and the increased clearance of $(S, S)$-darolutamide as compared with $(S, R)$-darolutamide in the presence of an AKR1C3 inhibitor in hepatocytes is attributed to the fact that this diastereomer is a more preferred substrate for oxidative metabolism as well as for glucuronidation.

Clearance of darolutamide by glucuronidation, leading to $O$-glucuronide M-7 isomers, is mainly mediated by UGT1A9, UGT1A1, and UGT1A3. $\mathrm{N}$-glucuronidation to form $\mathrm{M}-15$ isomers, however, appears to be mediated by UGT2B10 only, a primarily hepatic enzyme, which explains the lower amounts of glucuronides formed in other tissues (renal, intestinal) (Kaivosaari et al., 2011). Glucuronidation is a major elimination route of darolutamide, as the fraction metabolized via glucuronidation is at least one-third (assuming none of the darolutamide observed in feces was due to hydrolysis of glucuronides in the gut) or at most two-thirds (assuming darolutamide observed in feces was completely formed by hydrolysis of the glucuronides in the gut) (Fig. 12). Regarding the drug-drug interaction potential related to the glucuronidation pathway, the maximal effect of UGT inhibitors is theoretically predicted by a simplified static model to increase darolutamide exposure by a factor of 2.6-fold. In clinical studies, strong inhibition of the UGT1A9 substrate dapagliflozin, which has a similar metabolized fraction of 0.66 , with mefenamic acid increased the exposure of dapagliflozin mildly, by 1.5-fold (Kasichayanula et al., 2013; https://

\section{Excretion}

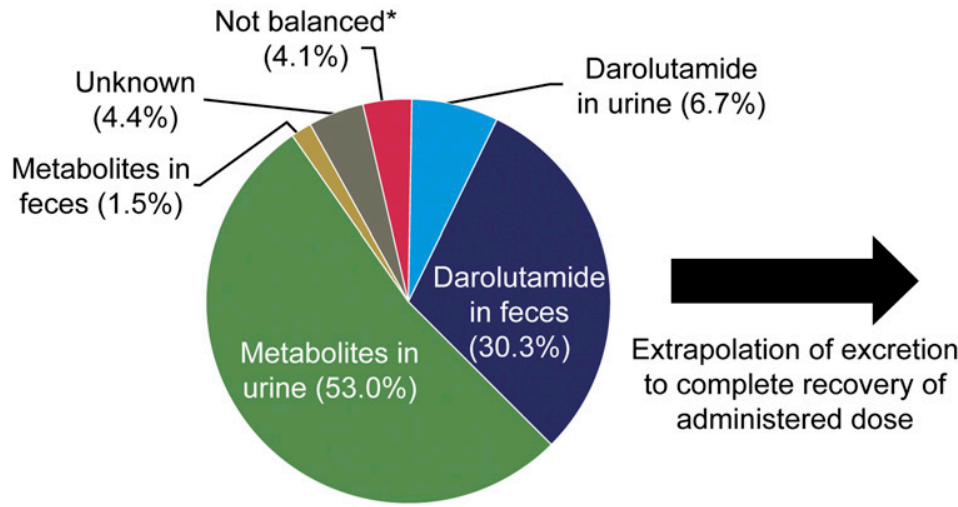

\section{Clearance}

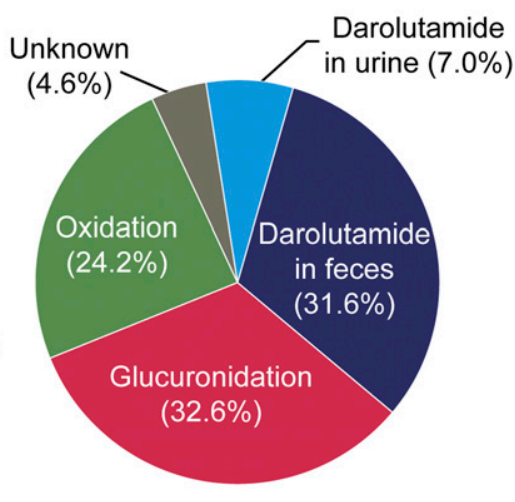

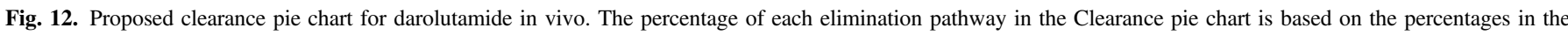
Excretion pie chart by excluding the not-balanced portion; data are the average of six subjects. *Not-balanced portion: difference between mean total $\mathrm{A}_{\mathrm{e}}$ (percentage of dose) in urine and feces and sum of all mean individual $\mathrm{A}_{\mathrm{e}}$ values per metabolite plus darolutamide recovered via radiochromatography. 
www.ema.europa.eu/en/documents/product-information/forxiga-eparproduct-information_en.pdf). Given this, and the likely compensation by the other clearance pathways for darolutamide (oxidation/reduction and direct secretion into the intestine), the effect on darolutamide exposure of coadministered UGT inhibitors is not expected to be clinically relevant. Although rifampicin, which can induce UGT1A9, decreased the exposure of darolutamide $\left(\mathrm{AUC}_{0-72}\right)$ by $72 \%$ after repeated coadministration (Zurth et al., 2019), this is far more likely to be due to the induction of CYP3A4, and possibly also P-glycoprotein, since the exposure of the sensitive UGT1A9 substrate, dapagliflozin, was only reduced by $22 \%$ by rifampicin (Kasichayanula et al., 2013). Thus, the effect of UGT inducers on darolutamide is unlikely to be clinically relevant.

Taken together, in vivo and in vitro data indicate that darolutamide is mainly cleared via formation of metabolites by oxidation and glucuronidation, with direct excretion of darolutamide also contributing. The proposed excretion and clearance scenario is summarized in Fig. 12 and shows three almost equally weighted elimination pathways: oxidation (24\%), glucuronidation (33\%), and excretion of darolutamide (feces $32 \%$, urine $7 \%$ ). CYP3A4 and AKR1C3 were identified as the main enzymes responsible for oxidative and reductive metabolism, respectively, whereas several UGT isoforms contributed to darolutamide glucuronidation. Since several pathways contribute to the overall clearance of darolutamide, the risk of relevant changes in the exposure of darolutamide because of the presence of potent inhibitors or inducers of UGT is likely to be low. Similarly, these several clearance pathways for darolutamide can mitigate the risk for changes in exposure with coadministration of CYP3A4 inhibitors, which is confirmed by clinical studies (Shore et al., 2019; Zurth et al., 2019).

\section{Acknowledgments}

The authors thank the participants and all investigators involved in this study. Stuart Mair of Quotient Clinical is acknowledged for conduct of the clinical part of the study. From Orion Pharma, Arja Vuorela is acknowledged for scientific support in the mass balance study, Ulla Sairanen for management of the clinical phase I study, Hanna Wartiovaara for supervision of the clinical bioanalytical work, Anne Luiro for radiosynthesis of ${ }^{14} \mathrm{C}$-darolutamide, and Olli Törmäkangas for the work to provide several unlabeled metabolites as structural references. From Bayer, Isabel Piel is acknowledged for isolation of several metabolites from human urine for structural identification and Christian Kaudelka for the synthesis of unlabeled M-36 as structural reference. Medical writing support, including assisting authors with the development of the outline and initial draft and incorporation of comments, was provided by Tamsin Williamson and Francesca Kolbe, and editorial support, including figure preparation, formatting, proofreading, and submission, was provided by Annabel Ola, Travis Taylor, and Beth King, all of Scion Medica, London, supported by Bayer HealthCare Pharmaceuticals, Inc. (Whippany, NJ), according to Good Publication Practice guidelines (https:// www.acpjournals.org/doi/10.7326/M15-0288). The sponsor was involved in the study design, collection, analysis, and interpretation of data, as well as data checking of information provided in the manuscript. However, ultimate responsibility for opinions, conclusions, and data interpretation lies with the authors.

\section{Authorship Contributions}

Participated in research design: Taavitsainen, Prien, Korjamo, Denner, Nykänen, Vuorela, Jungmann, von Bühler, Koskinen, Zurth, Gieschen.

Conducted experiments: Taavitsainen, Prien, Kähkönen, Korjamo, Denner, Nykänen, Vuorela, Jungmann, von Bühler, Koskinen, Zurth, Gieschen.

Performed data analysis: Taavitsainen, Prien, Kähkönen, Niehues, Korjamo, Denner, Nykänen, Vuorela, Jungmann, von Bühler, Koskinen.

Wrote or contributed to the writing of the manuscript: Taavitsainen, Prien, Kähkönen, Niehues, Korjamo, Denner, Nykänen, Vuorela, Jungmann, von Bühler, Koskinen, Zurth, Gieschen.

\section{References}

Brater DC (2002) Measurement of renal function during drug development. Br J Clin Pharmacol 54:87-95.

Fizazi K, Shore N, Tammela TL, Ulys A, Vjaters E, Polyakov S, Jievaltas M, Luz M, Alekseev B, Kuss I, et al.; ARAMIS Investigators (2019) Darolutamide in nonmetastatic, castration-resistant prostate cancer. N Engl J Med 380:1235-1246.

Fizazi K, Shore N, Tammela TL, Ulys A, Vjaters E, Polyakov S, Jievaltas M, Luz M, Alekseev B, Kuss I, et al.; ARAMIS Investigators (2020) Nonmetastatic, castration-resistant prostate cancer and survival with darolutamide. $N$ Engl J Med 383:1040-1049.

Gibson CR, Lu P, Maciolek C, Wudarski C, Barter Z, Rowland-Yeo K, Stroh M, Lai E, and NicollGriffith DA (2013) Using human recombinant UDP-glucuronosyltransferase isoforms and a relative activity factor approach to model total body clearance of laropiprant (MK-0524) in humans. Xenobiotica 43:1027-1036.

Kaivosaari S, Finel M, and Koskinen M (2011) N-glucuronidation of drugs and other xenobiotics by human and animal UDP-glucuronosyltransferases. Xenobiotica 41:652-669.

Kasichayanula S, Liu X, Griffen SC, Lacreta FP, and Boulton DW (2013) Effects of rifampin and mefenamic acid on the pharmacokinetics and pharmacodynamics of dapagliflozin. Diabetes Obes Metab 15:280-283.

Levey AS, Stevens LA, Schmid CH, Zhang YL, Castro AF III, Feldman HI, Kusek JW, Eggers P, Van Lente F, Greene T, et al.; CKD-EPI (Chronic Kidney Disease Epidemiology Collaboration) (2009) A new equation to estimate glomerular filtration rate. Ann Intern Med 150:604-612.

Massard C, Penttinen HM, Vjaters E, Bono P, Lietuvietis V, Tammela TL, Vuorela A, Nykänen P, Pohjanjousi P, Snapir A, et al. (2016) Pharmacokinetics, antitumor activity, and safety of ODM201 in patients with chemotherapy-naive metastatic castration-resistant prostate cancer: an openlabel phase 1 study. Eur Urol 69:834-840.

Moilanen AM, Riikonen R, Oksala R, Ravanti L, Aho E, Wohlfahrt G, Nykänen PS, Törmäkangas OP, Palvimo JJ, and Kallio PJ (2015) Discovery of ODM-201, a new-generation androgen receptor inhibitor targeting resistance mechanisms to androgen signaling-directed prostate cancer therapies. Sci Rep 5:12007.

Nykänen P, Korjamo T, Gieschen H, Zurth C, and Koskinen M (2020) Pharmacokinetics of darolutamide, its diastereomers and active metabolite in the mouse: response to Saini NK et al. (2020). Drug Metab Lett DOI: 10.2174/1872312814666201112121129 [published ahead of print].

Shore N, Zurth C, Fricke R, Gieschen H, Graudenz K, Koskinen M, Ploeger B, Moss J, Prien O, Borghesi G, et al. (2019) Evaluation of clinically relevant drug-drug interactions with darolutamide in the phase 3 ARAMIS trial for patients with nonmetastatic castration-resistant prostate cancer. Target Oncol 14:527-539.

Sugawara T, Baumgart SJ, Nevedomskaya E, Reichert K, Steuber H, Lejeune P, Mumberg D, and Haendler B (2019) Darolutamide is a potent androgen receptor antagonist with strong efficacy in prostate cancer models. Int J Cancer 145:1382-1394.

Taavitsainen P, Gieschen H, Korjamo T, Kähkönen M, Malmström C, Prien O, Niehues M Sandmann S, Janssen W, and Koskinen M (2020) Absorption, distribution, metabolism and excretion of darolutamide (a novel non-steroidal androgen receptor antagonist) in rats. Xenobiotica 50:967-979.

Zurth C, Koskinen M, Fricke R, Prien O, Korjamo T, Graudenz K, Denner K, Bairlein M, von Bühler C-J, Wilkinson G, et al. (2019) Drug-drug interaction potential of darolutamide: in vitro and clinical studies. Eur J Drug Metab Pharmacokinet 44:747-759.

Address correspondence to: Hille Gieschen, Bayer AG, Research and Development, Pharmaceuticals, 13342 Berlin, Germany. E-mail: hille.gieschen@ bayer.com 